\title{
Response time distributions: Some simple effects of factors selectively influencing mental processes
}

\author{
RICHARD SCHWEICKERT and MASS GIORGINI \\ Purdue University, West Lafayette, Indiana
}

\begin{abstract}
When hypotheses about mental processing are tested with response times, inferences are often based on means, and occasionally on variance or skewness. Calculations on entire distributions of response times are more informative and can be conveniently carried out. Recently investigators have been updating procedures primarily based on means (such as additive factors tests) to procedures employing entire distribution functions. In one such advance, Nozawa and Townsend upgraded earlier tests of whether factors selectively influence serial or parallel processes, and whether parallel processes enter AND gates or OR gates. We discuss generalizations of the tests to complex arrangements of processes in networks. Results for a particularly difficult network, the Wheatstone bridge, are presented here. We use simulations to demonstrate the feasibility of the tests, and the possibility of mimicking.
\end{abstract}

Much of what we know about mental processing has been deduced through detailed analysis of response times. One of the most successful keys to interpretation is the technique of selectively influencing individual process durations, pioneered by Sternberg (1969) in his additive factor method. If the processes required for performance of a task are in series, and if each of two experimental factors prolongs a different process, then the combined effect of both factors is predicted to be the sum of their individual effects. This simple test has had impact not only in its many applications (reviewed in Sanders, 1990), but also in the impetus it has given to the development of tests for further questions.

After some early setbacks caused by uncritical acceptance of unproven techniques, we are now able to answer a number of basic questions about process organization. The setbacks were due to the ability of conceptually different processing organizations to lead to mathematically identical predictions. An early influential paper by Townsend (1971) showed that serial and parallel process arrangements can make identical predictions about the effect on response time of the number of items to be processed. Another example is work by Marley and Colonius (1992) and Townsend (1976) showing that any model predicting choice response time and accuracy is equivalent to a parallel race of stochastically independent processes. Dzhafarov $(1993,1997)$ showed that any such model is equivalent to a system of deterministic processes racing for randomly preset criteria (the "Grice representability scheme," after Grice, 1968, 1970). To add to the difficul-

\footnotetext{
We give special thanks to Ehtibar Dzhafarov for unique insights and many helpful comments. We have benefited from discussions with Anli Lin, Richard A. Chechile, Georgie Nozawa, and James T. Townsend. Portions of this work were supported by NSF Grant 9123865 -DBS to R.S. Correspondence should be addressed to R. Schweickert, Psychological Sciences, 1364, Purdue University, West Lafayette, IN 479071364 (e-mail: swike@psych.purdue.edu).
}

ties, even when models are distinguishable mathematically, they may mimic each other's predictions statistically, as Van Zandt and Ratcliff (1995) recently pointed out.

Over the years, however, more tests have become available, and researchers have become more cautious in formulating the underlying assumptions of their models, so the possibilities for mimicking have become more limited and manageable. Work on the problem of inferring process organization has been carried out by more people than can be mentioned here. Readers interested in more details will find them in the surveys by Coles, Smid, Scheffers, and Otten (1995), Luce (1986), Massaro and Cowan (1993), Schweickert (1993), Townsend (1990a), and Townsend and Ashby (1983).

This paper continues the quest by describing a method for using cumulative distribution functions to simultaneously test whether experimental factors selectively influence sequential or concurrent components of complex networks of processes, and whether these networks involve AND or OR gates. This work follows on earlier related results by Nozawa (1992) and Townsend and Nozawa (1995).

Initially, hypotheses about the effects of experimental factors were tested with mean response times, but it is clear that more information is potentially available from cumulative distribution functions; for a variety of approaches, see Balakrishnan (1994); Colonius and Vorberg (1994); Dzhafarov (1992); Fisher and Goldstein (1983); Goldstein and Fisher (1991, 1992); Kounios (1993); Meyer, Yantis, Osman, and Smith (1984); Miller (1982); Shaked and Shanthikumar (1994); Stoyan (1983); Townsend (1990b); and Van Zandt and Ratcliff (1995). Two techniques are relevant here for examining cumulative distribution functions to recover processing architectures under the assumption of selective influence. One, developed by Ashby and Townsend (1980) and Roberts and Sternberg (1993), will be discussed in a later section, where it provides empirical support for our assumption of the stochastic independence of process durations. A generalization of this 
technique has been described in Dzhafarov and Schweickert (1995). The other technique, based on cumulative distribution contrasts, was developed by Nozawa (1992) and Townsend and Nozawa (1995). A generalization of this technique has been described in Schweickert, Giorgini, and Dzhafarov (in press) and is further developed here.

Analysis of cumulative distribution functions is useful because it is quite possible for predictions made about mean reaction times to be satisfied, while predictions about cumulative distribution functions are not. Further, as our simulations will demonstrate, cumulative distributions appear to be more promising than means for distinguishing process arrangements that mimic each other.

The organization of the rest of the paper is as follows. We explain what cumulative distribution contrasts can be used for, and what they are. Then we describe our assumed underlying architecture, directed acyclic task networks. We summarize the evidence for and against two ancillary assumptions made for tractability. We then present simulations demonstrating the behavior of cumulative distribution function contrasts for various process arrangements, in particular for a difficult network, the Wheatstone bridge. The paper ends with derivations of results specifically for a Wheatstone bridge.

Many experiments in cognitive psychology are designed to answer questions about underlying architecture. The problem of distinguishing between AND gates and OR gates is not as common as the problem of distinguishing between serial and parallel processing. But both gates appear in familiar models, AND gates in exhaustive processing models, and OR gates in self-terminating processing models and in race models. Here is an example of each kind of gate, and of how experimental factors were used to test for it.

In a study on visual search, Egeth and Dagenbach (1991) investigated whether search was serial or parallel, and whether it was self-terminating or exhaustive. If elements in the display are processed in parallel, then a selfterminating search stops as soon as any target is processed (i.e., with an OR gate). An exhaustive search stops after all elements have been processed (i.e., with an AND gate).

Egeth and Dagenbach (1991) tested predictions for the effects on mean reaction time of selectively influencing processes. For example, in their Experiment 1, subjects searched for a target in a two-letter display. A display contained two Xs, two Os, or an X and an O. Processing of a particular element was prolonged by degrading its visual quality. When the target was absent, the subject had to process both elements to determine that neither was a target. The mean reaction times behaved as predicted for a parallel search ending with an AND gate. ${ }^{1}$ When two targets were present, the subject had to process only one. The mean reaction times behaved as predicted for a parallel search ending with an OR gate. An analysis of cumulative distribution functions, in addition to means, could provide further evidence for (or against) these conclusions.
As another example of the class of architectures considered here, Kounios, Osman, and Meyer (1987) proposed a dual-process model of sentence verification in which a fast process (search of semantic memory) raced independently and in parallel with a slow process (logical computation). The response was determined by whichever process finished first (i.e., by an OR gate). Kounios (1993) proposed that the semantic relation between the subject and the predicate of a sentence would selectively influence the fast search process, while a categorical relation between subject and predicate would selectively influence the slow computation process.

In this example, the results were not exactly as predicted, and the original model was rejected and modified. Briefly, Kounios (1993) predicted that the fast reaction times of the distribution would be affected only by the semantic relation, and the slow ones only by the categorical relation. The predictions were satisfied only in part. Kounios concluded that the proposed dual processes were present, but that other specialized mechanisms were present also. Here, we note an additional problem for the original model. If the median reaction times reported are used as approximations for the means, they do not behave as predicted for parallel processes followed by an OR gate (see note 1). The cumulative distribution analysis described here could also be carried out; presumably the outcome would be further evidence for rejecting the original model, but this is not implied by the earlier results.

\section{CUMULATIVE DISTRIBUTION CONTRASTS}

The usual analysis of variance (ANOVA) test that two experimental factors have additive effects on response time can be thought of as a test whether certain sums and differences of mean response times-a contrast-equals 0 . (If the design is larger than a $2 \times 2$, there will be more than one contrast, one for each degree of freedom in the interaction.) Suppose two factors are manipulated in an experiment. Suppose each factor has two levels, 1 and 2, and let the response time when the first factor is at level $i$ and the second factor is at level $j$ be denoted $R T_{i j}$ - with mean $E R T_{i j}$. Then the statement that the factors have additive effects can be written

$$
E R T_{11}-E R T_{12}-E R T_{21}+E R T_{22}=0 .
$$

Nozawa (1992) and Townsend and Nozawa (1995) noted that a contrast similar to that on the left side of Equation 1 could be written in terms of cumulative distribution functions. Let $G_{i j}(t)$ be the cumulative distribution function (cdf) for the response time $R T_{i j}$ - that is, $G_{i j}(t)=P\left(R T_{i j} \leq t\right)$, the probability that $R T_{i j}$ is less than or equal to $t$. For each factor, higher level numbers correspond to higher means. Consider two parallel processes followed by a final process. Suppose two experimental factors selectively influence the two parallel processes, 
and the final process begins when both of the parallel processes have finished (an AND gate). If certain ancillary assumptions are met, then the following inequality holds at every time $t$,

$$
G_{11}(t)-G_{12}(t)-G_{21}(t)+G_{22}(t) \geq 0
$$

(Nozawa, 1992; Townsend \& Nozawa, 1995). In other words, a contrast of the same form as that for the mean response times is predicted to be nonnegative.

Equation 2 is based on the assumption that both the parallel processes must be finished before the final process begins. The sign of the contrast was shown by Nozawa and Townsend (1995) to reverse if the AND gate in the assumption above is replaced with an OR gatethat is, if the final process begins as soon as either (rather than both) of the two parallel processes terminates. With an OR gate, for all times $t$,

$$
G_{11}(t)-G_{12}(t)-G_{21}(t)+G_{22}(t) \leq 0 .
$$

For an intuition about why the sign changes, consider mean reaction times. With two parallel processes followed by an AND gate, if one process is very short changing the duration of the other will have a big effect. If one process is very long, changing the duration of the other will have little effect. With two parallel processes followed by an OR gate, the situation is reversed. If one process is very short, the duration of the other will have little effect. If one process is very long, the duration of the other will have a big effect.

The predicted inequalities above have been tested in experiments (Nozawa, 1992; Townsend \& Nozawa, 1995). Figure 1 (after Nozawa, 1992, Figure 8) illustrates the results of one experiment. The stimuli were two lightemitting diodes located above and below a fixation point. On a given trial, one diode, the other, or both, or neither was turned on. If a diode was turned on, it was at either low intensity or high intensity. There were two tasks. In the OR task, the subject was instructed to press a key for "yes" if one or the other or both diodes were turned on.
Another key was pressed for "no." In the AND task, the response was "yes" if both diodes were turned on.

The figure shows cdf contrasts for the OR task and the AND task for "yes" responses when both diodes were turned on. The indices 11, 12, 21, and 22 in Equations 2 and 3 correspond to the four combinations of light intensity levels for the left and right diodes.

The contrasts were illustrated originally in Nozawa (1992) for survivor functions, rather than for cdfs, $G_{i j}(t)$. The survivor function at $t$ is simply one minus the cdf at $t$ - that is, $1-G_{i j}(t)$. Consequently, the predicted signs of the survivor contrasts are opposite to those given above for cdfs. Survivor functions are less familiar than cdfs, although they are more convenient for certain purposes.

The contrasts in Figure 1 have two striking features. First, each contrast preserves its sign throughout the time axis. Second, the signs are different for the OR task and the AND task - negative for OR, and positive for AND, as predicted by Townsend and Nozawa (1995).

Analysis of the data from individual subjects revealed an aspect of the processing that analysis of mean reaction time alone would not. Because the prediction is about the sign of the cdf contrast, the same sign is predicted for individual subjects as for data averaged over subjects. When subjects were considered individually, for many there was a small blip early in the curve, of the sign opposite to that predicted. The blip occurred at different times for different subjects, and hence did not appear in the aggregated data. The appearance of a blip in a subject's data was sufficient reason to reject a parallel model for that subject. The blip was explained by Townsend and Nozawa (1995) with a Poisson channel summation model, and the reader is referred to their paper for details.

Townsend and Nozawa (1995) showed that when processes in series are selectively influenced, the cdf (or survivor) contrast must have equal positive and negative areas. Their reasoning is as follows. The expected value of a positive random variable is the area under its survivor function (see, e.g., Cinlar, 1975). This means that
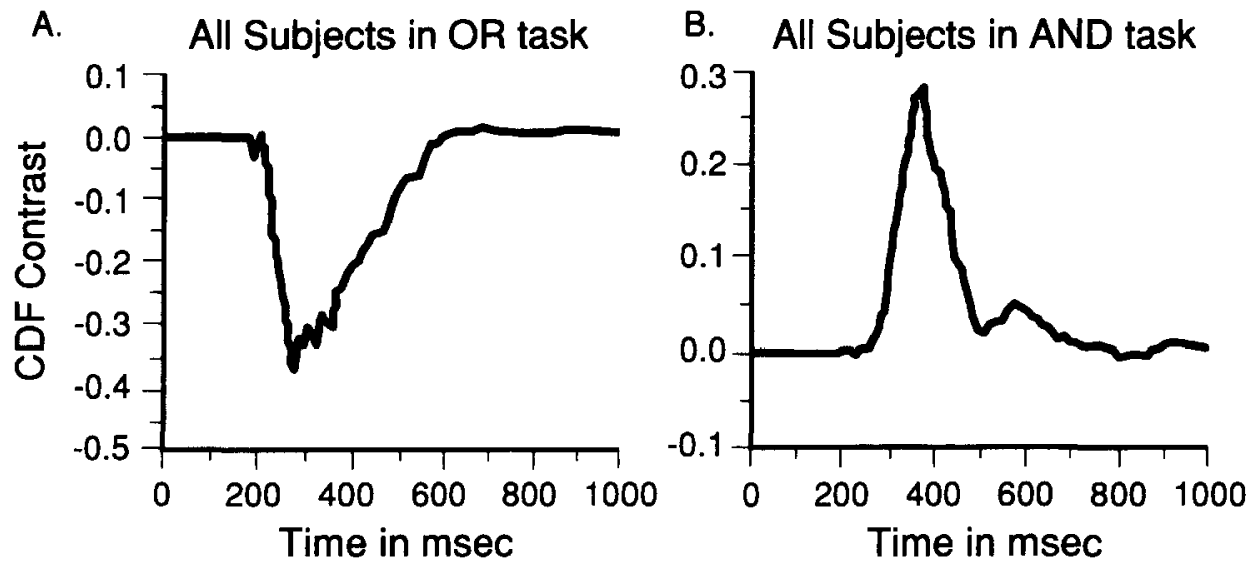

Figure 1. Response time cumulative distribution function (cdf) contrasts. These data are as predicted for selective influence of parallel processes. Each contrast has the same sign everywhere, but the sign is different for the OR gate and the AND gate. (After Nozawa, 1992, by permission.) 
$E R T_{i j}$ in Equation 1 is the area under $1-G_{i j}(t)$. It follows algebraically that the contrast of means

$$
E R T_{11}-E R T_{12}-E R T_{21}-E R T_{22}
$$

equals the area under

$$
\begin{aligned}
& 1-G_{11}(t)-\left(1-G_{12}(t)\right)-\left(1-G_{21}(t)\right)+1 \\
& -G_{22}(t)=-G_{11}(t)+G_{12}(t)+G_{21}(t)-G_{22}(t) .
\end{aligned}
$$

The mean contrast is zero for serial processes; hence the area under the survivor contrast must be zero.

The sign of the cdf contrast is opposite that of the survivor contrast, but the absolute value of the areas under the two curves is the same. Consequently, the area under the cdf contrast function when serial processes are selectively influenced is zero. Now that we have described cdf contrast functions, we will describe the architecture to be tested with them.

\section{Directed Acyclic Task Networks}

In this paper, we consider tasks with complex arrangements of processes. An example is a psychological refractory period task, in which a subject is presented with two stimuli, say, a light and a tone, and asked to respond to each, with, say, a buttonpress to identify the light and a spoken word to identify the tone. Such dual tasks typically begin with concurrent peripheral processing of the stimuli, followed by sequential processing in a central mechanism, followed by response processing, which may again be concurrent. There are many examples: Carrier and Pashler (1995); Davis (1957); Ehrenstein, Schweickert, Choi, and Proctor (1997); Johnston, McCann, and Remington (1995); McCann and Johnston (1992); Meyer and Kieras (1997a, 1997b); Osman and Moore (1993); Pashler and Johnston (1989); Ruthruff, Miller, and Lachmann (1995); Schweickert (1983a); Van Selst and Jolicoeur (1994); and Welford (1952). For reviews, see Pashler (1998) and Schweickert and Boggs (1984). For a variety of further examples, see Liu (1996).

Figure 2 shows the process arrangement in a hypothetical task used in our simulations. The task begins with four concurrent processes ( $a, y, c$, and $z$ ). Some of these are followed by other processes; for example, $w$ follows $a$. If one process follows another, the two are said to be sequential. (More formal definitions of the terms concurrent and sequential will be given later.) An arrow is sometimes needed to indicate the order in which

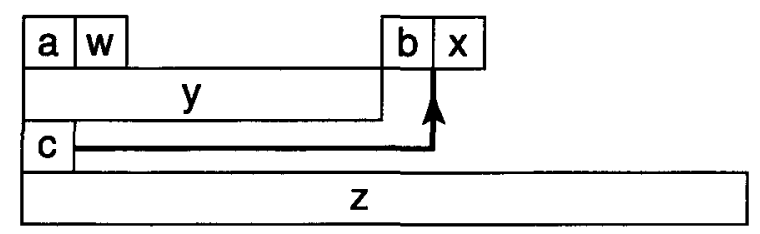

Figure 2. A task used in our simulations, represented as a bar chart. Some pairs of processes are concurrent; other pairs are sequential. the processes are carried out; for example, the arrow from process $c$ to process $x$ indicates that $c$ precedes $x$. Because a bar graph becomes hard to read if there are many such arrows, it is often preferable to abandon the attempt to use the length of the bars to represent the durations of the processes. One can simply represent each process with a node, all nodes the same size, and use arrows between nodes to indicate precedence. The result is a directed network. The task network in Figure 3 represents the same process arrangement as in Figure 2. Stimulus presentation is denoted by a starting node, $o$, of duration zero, and response onset is denoted by a terminal node, $r$, also of duration zero.

One of the purposes of this paper is to derive predictions for a network with a particularly difficult form, the Wheatstone bridge (Figures 4 and 5), which often arises in dual tasks. In Figure 4, two stimuli, $s 1$ and $s 2$, are presented, separated by a stimulus onset asynchrony (SOA), with responses $r 1$ and $r 2$, respectively. Each stimulus requires the execution of three processes, perception $(a)$, central processing $(b)$, and response preparation $(c)$. According to Welford's (1952) single-channel theory, the central processes for the two stimuli, $b 1$ and $b 2$, must be executed one at a time; hence in the model there is a process $s w$ for switching between $b 1$ and $b 2$.

One way a Wheatstone bridge will arise is if subjects are instructed to respond to the first stimulus before responding to the second, a common practice. This requirement is represented in the figure by an arrow directed from $r 1$ to $r 2$. The instruction that the responses be made in a particular order is not necessary to produce a Wheatstone bridge form. Models postulating a second, additional bottleneck - for example, postulating that $c 2$ be executed before $r 1$, also lead to a Wheatstone bridge as part of a larger network (see De Jong, 1993, Ehrenstein et al., (1997), Logan \& Burkell, 1986, and Schweickert, 1978, for double-bottleneck models).

What predictions can be made about the cdfs for such process arrangements? A number of predictions are easy to check graphically. Before describing them, we take a moment to consider the evidence for two ancillary assumptions sometimes made by Nozawa (1992) and Townsend and Nozawa (1995), because we will make these assumptions also.

\section{Support for Ancillary Assumptions}

Two ancillary assumptions are made for tractability. (1) Stochastic independence: The process durations are mutually stochastically independent, which means, loosely speaking, that the cdf for the duration of any process within the network does not depend on the durations of the remaining processes. More precisely, the joint cdf for any subset of the process durations is assumed to be the product of their corresponding marginal cdfs. (2) Stochastic dominance: When an experimental factor selectively influences a process, the cdf for the process duration at one level of the factor is assumed to be always greater than or equal to the cdf at another level of the factor. Pairs of 


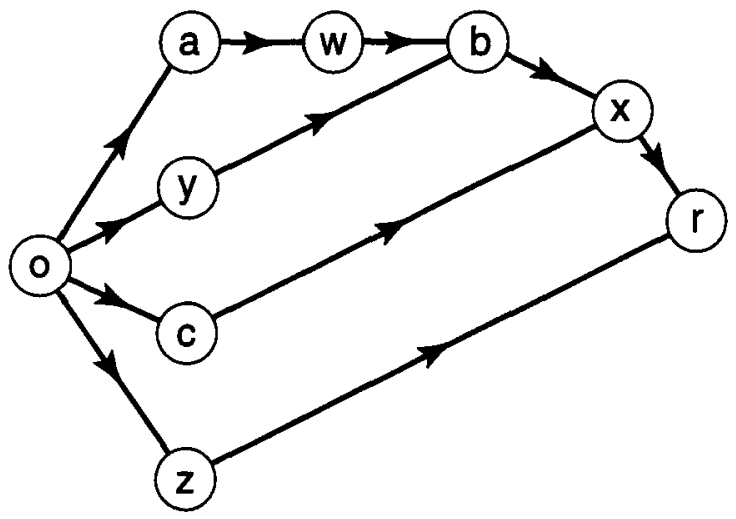

Figure 3. The same task as in Figure 2, represented here as a directed acyclic network. This is a serial-parallel network. In simulations, mean durations for the processes not manipulated were $o: 0, y: 300, z: 600, w: 50, x: 50$, and $r: 0$. Means of manipulated processes are shown in Tables 1 and 2.

cdfs with this property (or, more properly, the random variables underlying them) are said to be related by stochastic dominance. We say that the process corresponding to the upper cdf is stochastically faster than the other; in particular, its mean duration is smaller.

It is easy to check observable cdfs for stochastic dominance in a graph. Figure 6 (after Nozawa, 1992, Figure 4) illustrates stochastic dominance for the response time cdfs in the experiment mentioned above by Townsend and Nozawa (1995). The cdf for the response times to a single dot at low intensity lies above the corresponding cdf for high intensity. The functions do not cross at any point, although they may touch. In this section, we discuss the empirical support for these two assumptions, beginning with stochastic dominance.

Stochastic dominance. When an experimental factor selectively influences a process, we usually assume that the mean duration of the process increases as the factor levels increase-that is, as the difficulty increases. But our topic is the cumulative distributions, and the assumption that the means are ordered implies little about the distributions as a whole (see, e.g., Stoyan, 1983; Townsend, 1990a). Hence, we make the stronger assumption that the factor produces stochastic dominance.

Stochastic dominance for the process durations cannot be tested directly because the durations of the individual processes within a network are not observable other than through their contribution to the overall response time. However, it is reasonable to expect that stochastic dominance for the individual process durations implies stochastic dominance for the response times, in the following sense. Let the process selectively influenced by a Factor A be stochastically faster at Level 1 than Level 2. Then we should expect that the response time at Level 1 will be stochastically faster than at Level 2, whatever the levels of the remaining factors influencing the response time. In particular, considering
$R T_{i j}$ in a $2 \times 2$ design, if the process selectively influenced by Factor $\mathrm{A}$ is stochastically faster at $i=1$ than at $i=2$, and if the analogous assumption is true for $j=1$ and $j=2$, then for every time $t$ we expect

$$
\begin{aligned}
G_{11}(t) & \geq G_{12}(t) \\
G_{12}(t) & \geq G_{22}(t) \\
G_{11}(t) & \geq G_{21}(t) \\
G_{21}(t) & \geq G_{22}(t) .
\end{aligned}
$$

These predictions can be rigorously proved for any serial-parallel network, such as the one in Figure 3 (Schweickert et al., in press). The method used in those proofs does not apply to the Wheatstone bridge (Figure 5). In this paper we use a different approach to derive the predictions for the Wheatstone bridge (see the Appendix). In other words, if the network is assumed to be serial-parallel, or the Wheatstone bridge in Figure 5, then response time cdfs can falsify the assumption of stochastic dominance for the process durations. For networks for which a Wheatstone bridge is only a part, we lack a mathematical proof. We propose as a working hypothesis that the property in question holds for all networks; that is, sto-

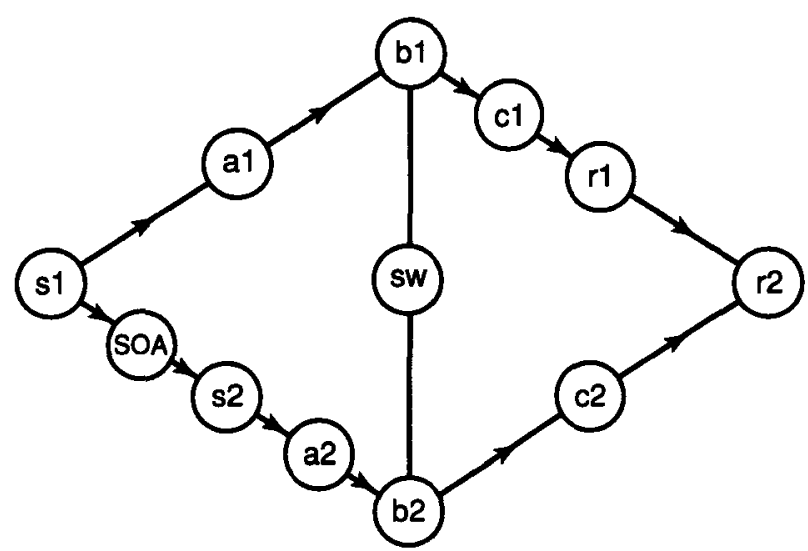

Figure 4. A directed acyclic network model for a dual task, with a Wheatstone bridge shape.

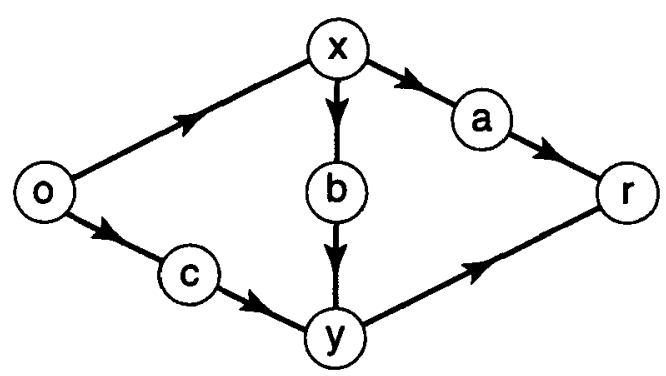

Figure 5. The Wheatstone bridge used in our simulations. A directed acyclic network is serial-parallel if and only if it does not contain a Wheatstone bridge. In simulations, mean durations were $o: 0, a: 250, b: 10, c: 500$, and $r: 0$. Means of $x$ and $y$ are in Table 3. 


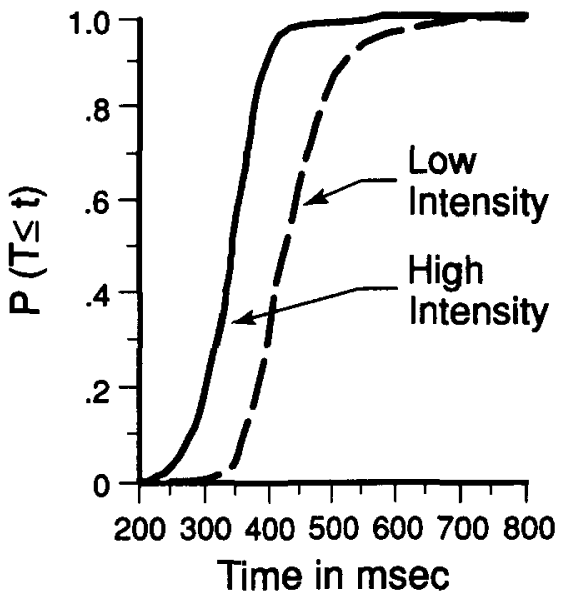

Figure 6. The response time cumulative distribution function (cdf) for low intensity lies below that for high intensity. The data exhibit stochastic dominance. (After Nozawa, 1992, by permission.)

chastic dominance for selectively influenced component durations implies stochastic dominance for overall response times. With this hypothesis, there seems to be considerable evidence for stochastic dominance in the literature.

Investigators occasionally publish graphs of the cdfs for response times at various levels of a factor, and when such graphs have been reported, stochastic dominance tends to be found. The data reported in Nozawa (1992) and Townsend and Nozawa (1995) are an example. Here are four more examples: (1) In a memory scanning task, Ashby, Tein, and Balakrishnan (1993, Figure 2) found that changing set size produced stochastic dominance of response times; in fact it occurred for every single subject. (2) In a dual-task experiment by Osman and Moore (1993, Figure 6) Response 1 was a keypress with the foot to a high or a low tone, and Response 2 was a keypress with a finger to a letter presented visually. Changing SOA over three levels produced almost perfect stochastic dominance for response times, for both responses. (3) In a simple response time experiment by Backus and Sternberg (1988, Experiment 1), a lever was pulled to respond to a light. The two factors of foreperiod duration and intensity produced stochastic dominance (see Roberts \& Sternberg, 1993, Figure 26.2, panel A). (4) In an identification experiment by Sternberg (1969, Experiment 5), digits were presented visually and subjects responded by saying a digit. Stimulus quality was changed by adding noise, and $\mathrm{S}-\mathrm{R}$ compatibility was changed by asking subjects to name the digit or to name its successor. Both factors produced stochastic dominance when the number of alternative stimuli was two and also when it was eight (see Roberts \& Sternberg, 1993, Figure 26.2, panels B and C). In these examples, one could argue that the investigators published such graphs only because a noteworthy pattern was found. Nonetheless, the variety of the tasks and ex- perimental factors suggests that stochastic dominance is a robust phenomenon.

Stochastic independence. As with the assumption of stochastic dominance, the stochastic independence of the individual process durations within a network cannot be tested directly, because the durations are not observable. We will summarize four different indirect approaches to testing stochastic independence. Results using three of these support the assumption, and results of the fourth do not. The tasks that have been investigated are quite different from each other, and the obvious implication is that stochastic independence occurs in some situations, but not in others. At first this conclusion may seem uninteresting, but stochastic independence is such a strong assumption that it is remarkable that it would ever be supported in a psychological task. For this reason, some results in Townsend and Nozawa (1995) were derived without assuming stochastic independence. For further discussion, see Balakrishnan (1994).

The first indirect test of stochastic independence is based on the fact that if process durations are stochastically independent, then factors selectively influencing different processes in series would not only have additive effects on the expected values of the response times, but also on the variances (Sternberg, 1969). In two identification experiments, Schwartz, Pomerantz, and Egeth (1977) tested for such additive effects on variance. In neither experiment was there a significant interaction, although it should be noted that in the second experiment the deviation from additivity was appreciable, albeit nonsignificant. Additive effects of factors on variances have also been found in four additional experiments summarized in Roberts and Sternberg (1993, Table 26.3).

The second indirect test is that if (1) process durations are stochastically independent and (2) the probability that the response is correct is the product of the probabilities that the individual processes are correct, then factors selectively influencing different serial processes would have additive effects not only on response time, but also on log percent correct (Schweickert, 1985). This prediction was confirmed in three studies previously reported in the literature-on memory scanning (Lively, 1972), lexical decision (Schuberth, Spoehr, \& Lane, 1981), and identification (Schwartz et al., 1977).

The third approach to testing stochastic independence is based on an analysis of cdfs. Because this approach is so closely related to the topic of this paper, we summarize it in some detail. Van Zandt and Ratcliff (1995) have given additional information about it, as well as a critical evaluation of the possibilities for mimicking.

The Ashby-Townsend-Roberts-Sternberg method is a test of whether the durations of two processes are stochastically independent and are combined serially to produce the response time. Suppose a subject performs a task by carrying out two serial processes $a$ and $b$, and suppose $a$ is finished before $b$ starts. Suppose one experimental factor selectively influences process $a$. Let 
the duration of process $a$ be the random variable $T_{a 1}$ when this factor is at Level 1 , and the random variable $T_{a 2}$ when this factor is at Level 2. Suppose another factor selectively influences process $b$, leading to durations $T_{b 1}$ and $T_{b 2}$, for Levels 1 and 2, respectively. The response time is the duration of process $a$ plus the duration of process $b$. When both factors are at Level 1 , the response time is $R T_{11}=T_{a 1}+T_{b 1}$, and so on.

Ashby and Townsend (1980) noted that by addition one can obtain two new random variables $R T_{11}+R T_{22}$ and $R T_{12}+R T_{21}$. The process durations forming the first sum, $\left(T_{a 1}+T_{b 1}\right)+\left(T_{a 2}+T_{b 2}\right)$, are a rearrangement of the process durations forming the second sum, $\left(T_{a 1}+\right.$ $\left.T_{b 2}\right)+\left(T_{a 2}+T_{b 1}\right)$. If the individual process durations are stochastically independent, then the two new random variables should have the same cdf. Ashby and Townsend proposed comparing estimates of the distributions of $R T_{11}+R T_{22}$ and of $R T_{12}+R T_{21}$. If these estimated distributions are equal, then the assumption of stochastic independence is supported indirectly, as is the assumption of seriality. (Contemporary methods for testing forms of distributions were recently reviewed by Chechile, 1997.)

Using a different, but logically equivalent technique, Roberts and Sternberg (1993) analyzed data from four experiments with the summation test. For each data set, empirical cdfs for $R T_{11}+R T_{22}$ and for $R T_{12}+R T_{21}$ were constructed with a special procedure. In each case, the two distributions were virtually identical. For readers interested in further work on this topic, a detailed analysis and a generalization of the Ashby-Townsend-RobertsSternberg test are given in Cortese and Dzhafarov (1995), Dzhafarov and Cortese (1995), and Dzhafarov and Schweickert (1995).

The fourth indirect test of stochastic independence of process durations was developed by Dzhafarov (1992) and elaborated by Dzhafarov and Rouder (1996). Simple response time to a signal with a step function onset can be decomposed as the sum of a component dependent on the signal and a component not dependent on the signal. Keypress responses to an abrupt change in location of a light source were collected. A distribution-free analysis of the response times, based on percentile ranks, revealed that both components could be written as increasing functions of the same random variable. This implies that the components were not stochastically independent.

To summarize, evidence that factors selectively influencing process durations produce stochastic dominance of the distribution functions for the process durations has been found for a wide assortment of factors in a variety of tasks. Stochastic independence of process durations appears to be less pervasive, although it too has been supported in a variety of tasks.

We turn now to predictions about cdf contrasts in tasks involving a mixture of sequential and concurrent processing. We will present results of simulations of factors selectively influencing processes in such a way that stochastic dominance was produced and stochastic independence was maintained.

\section{SIMULATIONS}

In our simulations, we supposed that a subject executed a set of mental processes arranged in the serialparallel network shown in Figure 3 or the Wheatstone bridge network of Figure 5. Each process is represented by a node and has a nonnegative duration that varies from trial to trial. If one process immediately precedes another, an arrow is drawn from the node representing the former process to the node representing the latter process. The task begins at a node, $o$, called the starting node, which indicates the presentation of the stimulus. No node precedes the starting node. Likewise, the task ends at a node, $r$, called the terminal node, which indicates the onset of the response. No node follows the terminal node.

A process $a$ precedes another process $b$ if there is a path starting at the node representing $a$, proceeding along some arc in the direction indicated by the arrow to another node, proceeding perhaps along further arcs and nodes, always in the directions indicated by the arrows, and ending at the node representing $b$. We say processes $a$ and $b$ are sequential if either $a$ precedes $b$ or $b$ precedes $a$. Two processes $a$ and $b$ are concurrent if they are not sequential.

If a process can begin execution when any one of its immediate predecessors has been completed, we say the process is released with an OR gate. If a process can begin execution only when all of its immediate predecessors have been completed, we say the process is released with an AND gate.

An AND gate or an OR gate can be constructed as follows with an accumulator, a mechanism that emits a signal when a counter reaches a preset criterion. Whenever a process immediately preceding the gate is completed, the counter is incremented by one. For an OR gate, the criterion is reached when the counter equals one. For an AND gate, the criterion is reached when the counter equals the number of processes immediately preceding the AND gate. When the criterion is reached, the gate sends a signal to start all the processes that immediately follow it.

We consider two types of networks here, those in which every gate is an OR gate and those in which every gate is an AND gate. The response is made at the terminal node, and we assume that the terminal node is an OR gate or an AND gate, of the same type as the others. Networks having both OR and AND gates, or with other types of gates, are possible, of course, but are beyond the scope of this paper.

In the simulations, all the gates were AND gates, so the response time was the sum of the durations of all the processes on the longest path through the network. The longest path through the network is called the critical path. A directed acyclic network with AND gates is sometimes called a critical path network or a PERT network. If all the gates are OR gates, the response time is the sum of the durations of all the processes on the shortest path from the starting node to the terminal node. 
In a network with AND gates or OR gates, a path directed from a node to itself would indicate that the process represented by the node would have to finish before it could start. This is impossible, so no process precedes itself, immediately or otherwise, and the task network is said to be a directed acyclic network. For more on task networks, see Fisher (1985), Fisher and Glasser (1996), and Schweickert (1978).

There are two kinds of directed acyclic task networks: (1) those that contain within them the Wheatstone bridge network illustrated in Figure 5 and (2) those that do not contain it (Dodin, 1985; Kaerkes \& Mohring, 1978). Directed acyclic networks not containing a Wheatstone bridge are called serial-parallel networks. Inspection of the network in Figure 3 shows that it does not contain as one of its parts the network in Figure 5, so Figure 3 is a serial-parallel network.

The simulations to be presented show that patterns in the cdfs for overall response times can be used to test between serial-parallel networks with AND gates and those with OR gates, and, within either type of serialparallel network, between sequential processes and concurrent processes. In a sense, this means that the serialparallel networks with selectively influenced components and with all AND gates or all OR gates are completely characterizable.

The Wheatstone bridge, and networks containing it, are more difficult. The simulations show that the sequential processes $x$ and $y$ in the Wheatstone bridge network of Figure 5 can produce the same response time patterns as either concurrent processes or sequential processes in a serial-parallel network - for a given $2 \times 2$ design. Employing a wide range of levels for each of the factors, however, allows one to identify sequential processes in a Wheatstone bridge. Results of our simulations suggest that cdfs are more effective than mean reaction times for distinguishing processes in a Wheatstone bridge from concurrent processes.

The simulations also show that regular patterns can be made manifest in the cdf contrasts with a feasible number of trials (2,000 per condition). The patterns appearing in the cdf interaction contrasts in these simulations lead us to consider whether they occur in general. We were able to prove that the patterns found in our simulations of the serial-parallel network generally occur (Schweickert et al., in press). The method of proof in that paper does not extend to networks that are not serial-parallel. For the Wheatstone bridge in Figure 5, derivations in the Appendix of this paper show that certain patterns generally occur, although general results for the most difficult case-processes $x$ and $y$ on opposite sides of the bridgeremain to be found.

Networks for which a Wheatstone bridge is only a part also remain an open question. Fortunately, certain networks containing Wheatstone bridges can usefully be reduced to serial-parallel networks. To explain how to do this, we need to define a module, a set of nodes that can be replaced with a single node. In Figure 4, SOA, $s 2$, and $a 2$ are an example. A module (or partitive set) is a set $M$ of nodes such that (1) if a node outside $M$ precedes any node of $M$, then it precedes all nodes of $M$; (2) if a node outside $M$ follows any node of $M$, then it follows all nodes of $M$; and (3) if a node outside $M$ is concurrent with any node of $M$, then it is concurrent with all nodes of $M$ (Schweickert, 1983b). A module can usually be replaced by a single node without loss of information. The exception occurs when the module happens to contain both of the processes selectively influenced by the factors; in that case, replacing the module with a single node loses the information about whether the selectively influenced processes are sequential or concurrent.

It may be that all the Wheatstone bridges in a network are contained in modules, none of which contains both of the processes selectively influenced by the factors. When each of the modules is replaced by a single node, the resulting reduced network may be a serial-parallel network. Then our results for serial-parallel networks would apply. However, such a reduction may not be possible. For example, the entire Wheatstone bridge in Figure 5 forms a module. But if processes $x$ and $y$ are selectively influenced, the Wheatstone bridge cannot be reduced to a single node without losing the information that $x$ and $y$ are sequential.

The following procedure was used for simulations of the networks in Figures 3 and 5. Each process has a nonnegative duration, which varies from trial to trial. The process durations were assigned independent normal distributions, truncated to yield nonnegative values. The mean of each process duration (prior to truncation) is given in the captions of Figures 3 and 5. For the specific processes, which were prolonged, additional mean durations were used, as given in Tables 1,2, and 3. The standard deviation of a process, prolonged or not, was set to one quarter of its mean (again, prior to truncation). Therefore, the distributions of the process durations are entirely characterized by their means. With this procedure, as the mean duration of a process increases, so does its standard deviation. This ensures that the standard deviation of the observable response time tends to increase with its mean (see, e.g., Luce, 1986). Further, since the distribution shape is fixed, the procedure produces stochastic dominance for the prolonged processes (Schweickert, Fisher, \& Goldstein, 1992). For each condition (i.e., combination of factor levels), 2,000 trials were simulated

Table 1

Simulated Response Time Means and Standard Deviations: Concurrent Processes $a$ and $c$ in Serial-Parallel Network of Figure 3

\begin{tabular}{ccccc}
\hline & \multicolumn{3}{c}{ Duration of Process $c$} \\
\cline { 2 - 5 } Duration of & \multicolumn{2}{c}{$c=50$} & & \multicolumn{2}{c}{$c=600$} \\
\cline { 2 - 5 } Process $a$ & $M$ & $S D$ & $M$ & $S D$ \\
\hline$a=50$ & 616 & 135 & 719 & 122 \\
$a=500$ & 706 & 110 & 753 & 101 \\
\hline
\end{tabular}

Note-In the simulations reported in this table, the mean of process $b$ was always 50 . 
Table 2

Simulated Response Time Means and Standard Deviations: Sequential Processes as $a$ and $b$ in Serial-Parallel Network of Figure 3

\begin{tabular}{ccccc}
\hline & \multicolumn{3}{c}{ Duration of Process $b$} \\
\cline { 2 - 5 } Duration of & \multicolumn{2}{c}{$b=50$} & \multicolumn{2}{c}{$b=500$} \\
\cline { 2 - 5 } Process $a$ & $M$ & $S D$ & $M$ & $S D$ \\
\hline$a=50$ & 719 & 122 & 882 & 124 \\
$a=500$ & 753 & 101 & 1,107 & 167 \\
\hline
\end{tabular}

Note-In the simulations reported in this table, the mean of process $c$ was always 600 .

using MICROSAINT (Micro Analysis and Design, 1985), using a different random number seed for each condition.

To see how the behavior of processes on opposite sides of a Wheatstone bridge differs from the behavior of other processes, one needs to see how processes not in a Wheatstone bridge behave. To this end, we first consider serialparallel networks.

\section{Serial-Parallel Networks}

For the network in Figure 3, two $2 \times 2$ designs were simulated, one for the pair of concurrent processes $a$ and $c$ (keeping the mean of process $b$ at 50), and the other for the sequential pair $a$ and $b$ (keeping the mean of process $c$ at 600). This means that in the first simulation, two experimental factors were assumed to selectively influence the concurrent processes $a$ and $c$, yielding four pairs of (truncated) independent normal distributions for their durations, while the distributions of the other processes remained fixed throughout the simulation. Analogously, in the second simulation two experimental factors were assumed to selectively influence the sequential processes $a$ and $b$, while the distributions of the other processes remained fixed.

Concurrent processes. The means and standard deviations of the simulated response times for the conditions in which the concurrent processes $a$ and $c$ were selectively influenced are shown in Table 1. The table shows clearly that there is a negative interaction for the meansthat is, $616-719-706+753=-56<0$. This negative interaction pattern in the means for concurrent processes agrees with theoretical predictions (Schweickert, 1978).

An analogous interaction can be calculated at each point in time using the cdfs rather than the means. The cdfs for the response times corresponding to the four different combinations of factor levels can be characterized by the mean durations of processes $a$ and $c$ :

$$
\begin{aligned}
& G_{11}(t)=G_{a 50, c 50}(t) \\
& G_{12}(t)=G_{a 50, c 600}(t) \\
& G_{21}(t)=G_{a 500, c 50}(t) \\
& G_{22}(t)=G_{a 500, c 600}(t) .
\end{aligned}
$$

The cdfs for the four conditions are shown in Figure 7, in the order (from left to right) $G_{a 50, c 50}(t), G_{a 500, c 50}(t)$,
$G_{a 50, c 600}(t), G_{a 500, c 600}(t)$. (The two intermediate curves practically coincide.)

The left-most curve is the cdf for the cell in which the processes being influenced are at their shortest durations used within the $2 \times 2$ design. The right-most curve is the cdf for the cell in which the two processes are at their longest. The intermediate curves are the cdfs for the cells in which one process was prolonged while the other was not.

The first step is to check that the cdfs satisfy stochastic dominance (Equation 4) - that is, that the left-most curve does not completely cross either of the intermediate ones, and neither of the intermediate curves completely crosses the right-most one. (It does not matter whether the two intermediate curves cross or not.) If this ordering condition were not satisfied by the cdfs in Figure 7 , further tests would not be applicable. Since the condition is satisfied, we proceed with the tests.

The next step is to calculate the interaction contrast from the cdfs. The cdf interaction contrast at each time $t$ is

$$
\begin{aligned}
c(t)= & G_{a 50, c 50}(t)-G_{a 50, c 600}(t)-G_{a 500, c 50}(t)+ \\
& G_{a 500, c 600}(t)
\end{aligned}
$$

(see Equation 2). It is shown in Figure 8. It has a curiously narrow peak, as do others we report. The most important finding is that this function is nonnegative at every moment of time. As we show in another study (Schweickert et al., in press), this sign invariance is characteristic for all serial-parallel networks with AND gates and independent process durations, in which the selectively influenced processes are concurrent. We show in the same study that if the network had OR gates rather than AND gates, the contrast would have the opposite sign; in this case, it would be nonpositive at all moments of time.

Sequential processes. The means and standard deviations of the simulated response times for the sequential processes $a$ and $b$ are shown in Table 2. (Parameter values for one cell in Table 2, $a=50, b=50, c=600$, are the same as those for a cell in Table 1, and the same simulated trials were used in both cases.) For the sequential processes, there is a positive interaction for the means, $719-753-882+1107=191>0$; that is, the mean contrast has the opposite sign to the one found in the previous simulation for concurrent processes. This agrees with theoretical predictions (see Schweickert, 1978;

\begin{tabular}{|c|c|c|c|c|c|c|}
\hline \multirow{3}{*}{$\begin{array}{l}\text { Duration of } \\
\text { Process } x\end{array}$} & \multicolumn{6}{|c|}{ Duration of Process $y$} \\
\hline & \multicolumn{2}{|c|}{$y=25$} & \multicolumn{2}{|c|}{$y=100$} & \multicolumn{2}{|c|}{$y=600$} \\
\hline & $M$ & $\overline{S D}$ & $M$ & $S D$ & $M$ & $S D$ \\
\hline$x=50$ & 527 & 117 & 600 & 124 & 1,101 & 194 \\
\hline$x=200$ & 555 & 101 & 610 & 111 & 1,097 & 187 \\
\hline$x=800$ & 1,056 & 204 & 1,061 & 200 & 1,426 & 233 \\
\hline
\end{tabular}
Schweickert et al., 1992; Schweickert \& Townsend, 1989; Schweickert \& Wang, 1993).

Table 3

Response Time Means and Standard Deviations: Sequential Processes $x$ and $y$ in Wheatstone Bridge Network of Figure 5 


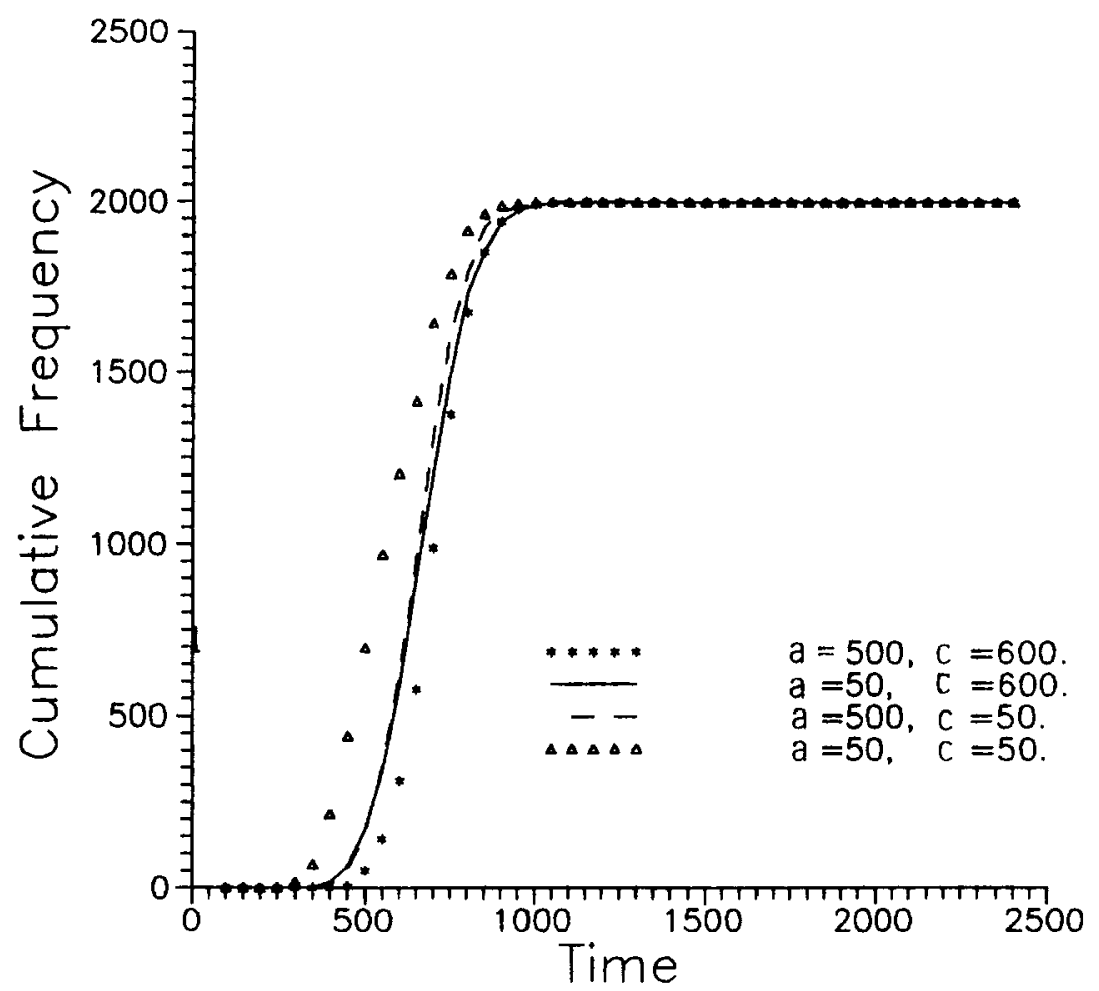

Figure 7. Simulated response time cumulative distribution functions (cdfs) for the concurrent processes $a$ and $c$ in Figure 3. The cdfs exhibit stochastic dominance.

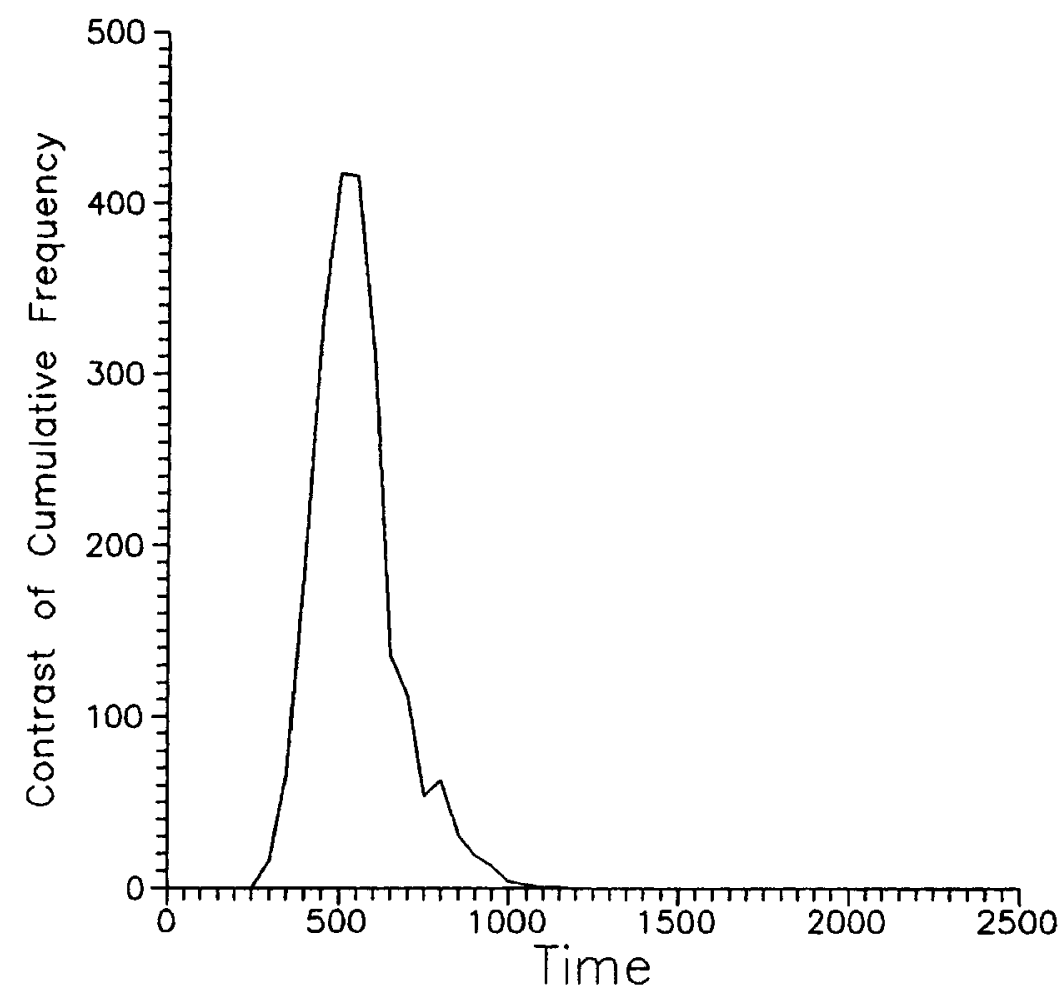

Figure 8. Simulated cumulative distribution function (cdf) interaction contrast (Inequality 2) for the concurrent processes $a$ and $c$ in Figure 3, with AND gates. 


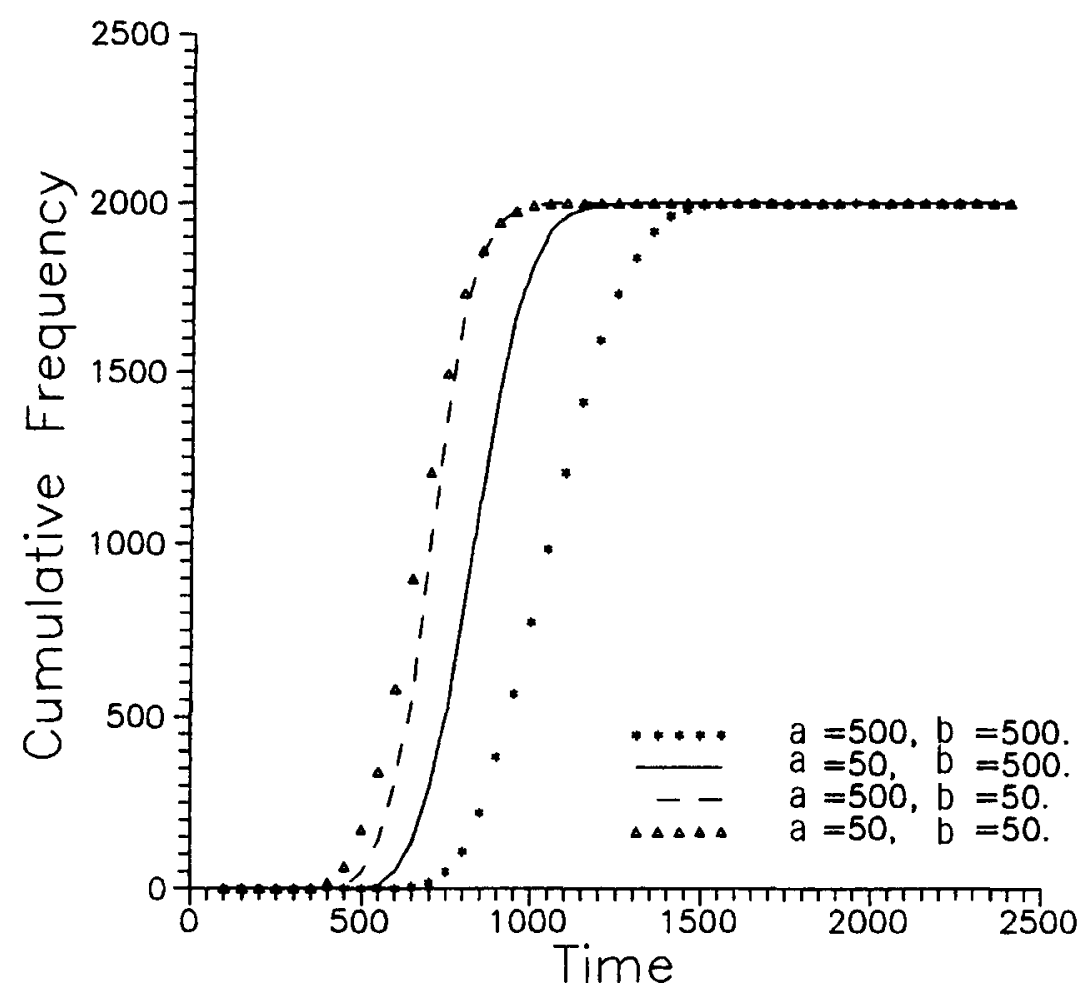

Figure 9. Simulated response time cumulative distribution functions (cdfs) for the sequential processes $a$ and $b$ in Figure 3 . The cdfs exhibit stochastic dominance.

As before, the analogous contrast can be formed with cdfs. We use the following notation here:

$$
\begin{aligned}
& G_{11}(t)=G_{a 50, G b 50}(t) \\
& G_{12}(t)=G_{a 50, b 500}(t) \\
& G_{21}(t)=G_{a 500, b 50}(t) \\
& G_{22}(t)=G_{a 500, b 500}(t) .
\end{aligned}
$$

The cdfs of Equation 6 are shown in Figure 9, in the order (from left to right) $G_{a 50, b 50}(t), G_{a 500, b 50}(t), G_{a 50, b 500}(t)$, $G_{a 500, b 500}(t)$. Once again, the prerequisite for further testing is that the cdfs satisfy stochastic dominance, which they do.

The cdf contrast is calculated in the same manner as for concurrent processes, $G_{a 50, b 50}(t)-G_{a 50, b 500}(t)-$ $G_{a 500, b 50}(t)+G_{a 500, b 500}(t)$. In Figure 10, the contrast can be seen to have the following two properties: (1) It is positive for low values of $t$ but becomes negative later. (2) The net area bounded by the entire curve and the axis at 0 is negative; in fact, the net area from any time $t$ to infinity is negative. The cdf contrast is strikingly different for concurrent and sequential processes. The most conspicuous difference is that the curve for concurrent processes does not change sign, while the curve for sequential processes does change signs.

In general, for sequential processes in a serial-parallel network, with AND gates, (1) the cdf interaction contrast is positive (or 0 ) for a period of time after time 0 , and then becomes negative (or 0 ). It may change signs more than once. (2) The net area bounded by the cdf contrast curve is negative (or 0 ) from any arbitrary time $t$ to infinity. Statement 1 is also true if the gates are OR gates, but Property 2 is modified as follows: The net area bounded by the cdf contrast curve is positive (or 0 ) from 0 to any arbitrary time $t$ (Schweickert et al., in press). The key characteristics are summarized in Table 4. Note that if a contrast changes to 0 from, say, positive, no sign change will be evident.

It is worth noting, at this point, that distinguishing networks with AND gates from those with OR gates is relatively straightforward provided that a factor can be found to selectively influence a process in such a way that it has an effect on response time at some levels but not at other levels. Consider a factor selectively influencing a process $a$, and suppose some other process $z$ is concurrent with $a$. For simplicity, suppose $a$ and $z$ are the only two processes, and $z$ takes 5 units of time. If the gates are AND gates, then when the duration of $a$ is long, $a$ will be on the critical path, so changing the duration of $a$ will have an effect on response time. However, when the duration of $a$ is short, say 3 units, $a$ may not be on the critical path, so small changes in the duration of $a$ may not have an effect on response time. In other words, at 


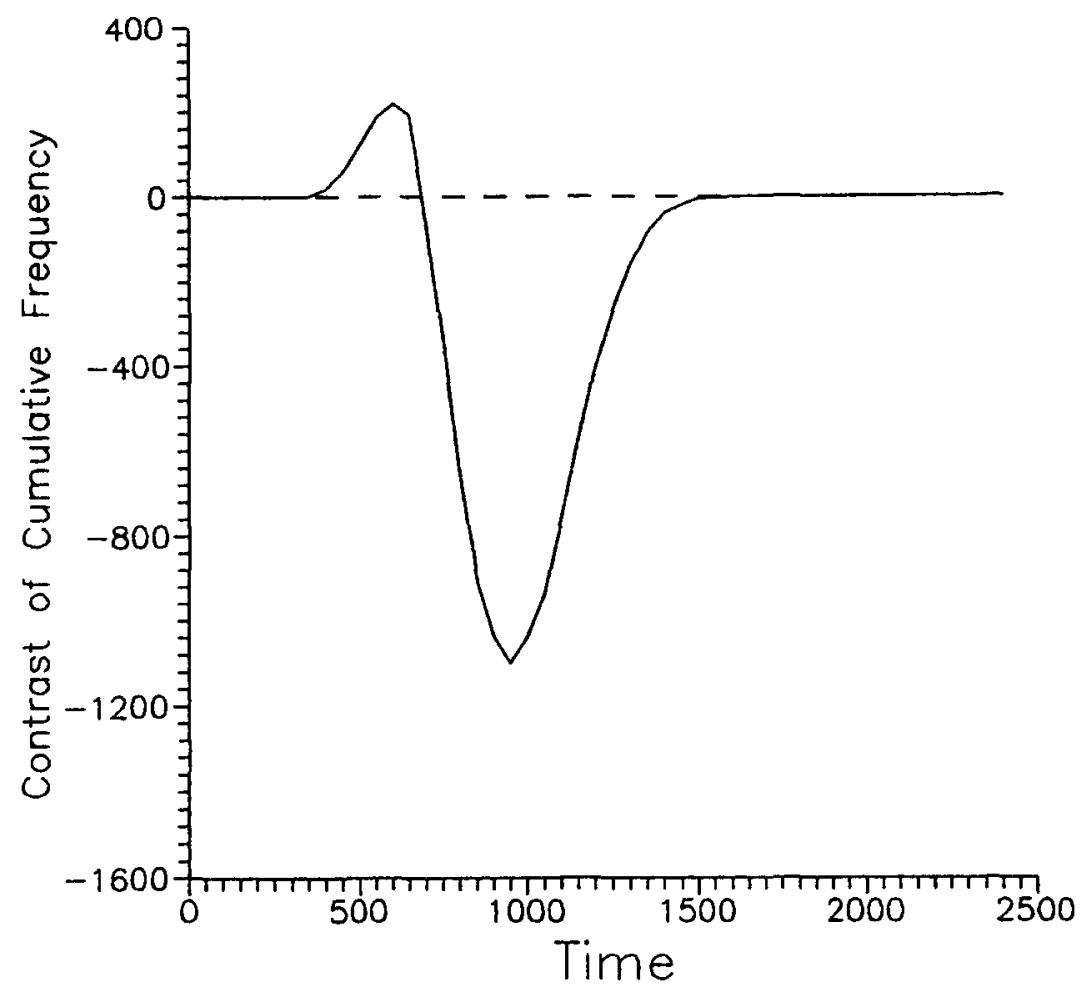

Figure 10. Simulated cumulative distribution function (cdf) interaction contrast for the sequential processes $a$ and $b$ in Figure 3, with AND gates.

low levels of the factor, changing the levels of the factor may have no effect on reaction time, but at high levels changing levels will have an effect.

The situation is just the opposite if the gates are OR gates. In that case, a small change in factor levels will have an effect on response time when the original factor level is small. But a small change in factor levels will have no effect when the original factor level is large.

(If a change in factor levels has an effect on response time at all levels of the factor influencing $a$, then process $a$ is not relevant for this procedure for resolving the question about the gates, which must be settled by considering some other process. If no other process is relevant, then there are no gates in the network.)

Once it is determined whether the gates are AND gates or OR gates, the patterns in the cdf contrast distinguish concurrent from sequential processes if the network is a serial-parallel network (Table 4). One potential difficulty is that although the cdf contrast for sequential processes typically changes sign at least once, it need not do so. However, Property 2 requires that a nonzero cdf contrast for sequential processes that does not change sign must be negative with AND gates and positive with OR gates. These signs are opposite to those predicted for concurrent processes. Hence, sequential and concurrent processes would not be confused even when the cdf contrast does not change sign, once the type of gate is known.

\section{The Wheatstone Bridge}

It follows from this discussion that if the network of mental processes is known (or assumed) to be serialparallel, and if the assumptions of stochastic dominance, stochastic independence, and selective influence are met, then one can readily decide whether the two processes selectively influenced are concurrent or sequential.

Theoretical results for pairs of processes in the Wheatstone bridge in Figure 5 are derived in the Appendix. Most results are the same as for serial-parallel networks. That is, for all the concurrent pairs, and all but one of the sequential pairs, the conclusions drawn earlier for serial-

Table 4

Characteristics of Cumulative Distribution Function Contrasts

\begin{tabular}{lcc}
\hline & \multicolumn{2}{c}{ Gates } \\
\cline { 2 - 3 } Arrangement & AND & OR \\
\hline Processes in Serial-Parallel Networks or in the Wheatstone Bridge \\
(but Not on Opposite Sides of the Bridge)
\end{tabular}

Note-Contrasts equal to 0 at all times $t$ are ignored in this table. 
parallel networks are true for the Wheatstone bridge as well. The exception is the pair of sequential processes on opposite sides of the "bridge" - that is, $x$ and $y$ in Figure 5 . The pattern of the cdf contrast revealed in the simulations is more complicated, although still systematic, for these processes. We begin our discussion by considering the results of our simulations.

The simulations were carried out for a $3 \times 3$ design with two factors selectively influencing the sequential processes $x$ and $y$ in Figure 5. The procedure was the same as in the previous simulations. For the cdfs, we consider two $2 \times 2$ subdesigns of the $3 \times 3$ design in Table 3 . The first subdesign is made of the conditions with the smallest and intermediate means; the second is made of the conditions with the intermediate and largest means.

In the first subdesign, the factor levels correspond to the stochastically fastest and intermediate processes. The cdf contrast is

$$
G_{x 50, y 25}(t)-G_{x 50, y 100}(t)-G_{x 200, y 25}(t)+G_{x 200, y 100}(t) \text {. }
$$

In the second design, the factor levels correspond to the stochastically intermediate and slowest processes. The cdf contrast is

$G_{x 200, y 100}(t)-G_{x 200, y 600}(t)-G_{x 800, y 100}(t)+G_{x 800, y 600}(t)$.

The cdfs for the two subdesigns are shown in Figures 11 and 12 , respectively, and the corresponding cdf con- trasts are shown in Figures 13 and 14, respectively. Inspection of Figures 11 and 12 shows that the cdfs satisfy stochastic dominance.

However, the cdf contrast patterns for the two subdesigns are markedly different. The cdf contrast in Figure 13 is nonnegative at all moments of time. By comparing this figure with Figure 8, one can see that the sequential processes in the Wheatstone bridge are capable of producing the sign invariance characteristic of concurrent processes in a serial-parallel network. But, as Figure 14 shows, the cdf contrast for the second $2 \times 2$ subdesign does change sign, producing the sign change characteristic of sequential processes in a series-parallel network (cf. Figure 10).

The mark of a Wheatstone bridge. It is not easy to distinguish experimentally between the sequential processes on opposite sides of a Wheatstone bridge (i.e., $x$ and $y$ in Figure 5) and concurrent processes. One approach is with mean reaction times. Unfortunately, the sign of the mean interaction contrast is not informative. For factors selectively influencing concurrent processes in an AND network, the mean interaction contrast is predicted to be negative (Schweickert, 1978, 1982). The same is true for factors selectively influencing the sequential processes on opposite sides of a Wheatstone bridge (Schweickert, 1982; Schweickert et al., 1992; Schweickert \& Townsend, 1989; Schweickert \& Wang, 1993).

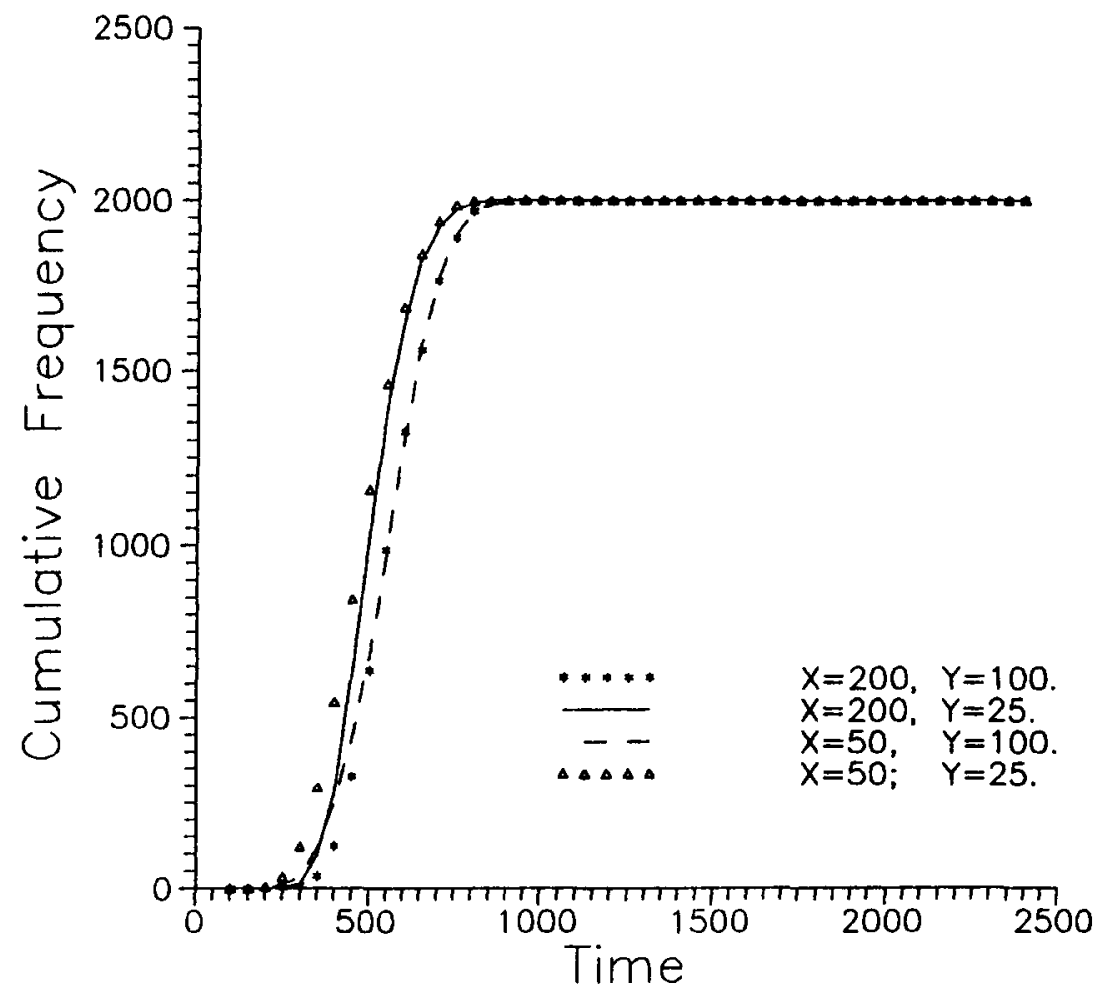

Figure 11. Simulated response time cumulative distribution functions (cdfs) for the sequential processes $x$ and $y$ in the Wheatstone bridge of Figure 5. The cdfs exhibit stochastic dominance. Prolongations of $x$ and $y$ are relatively small. 


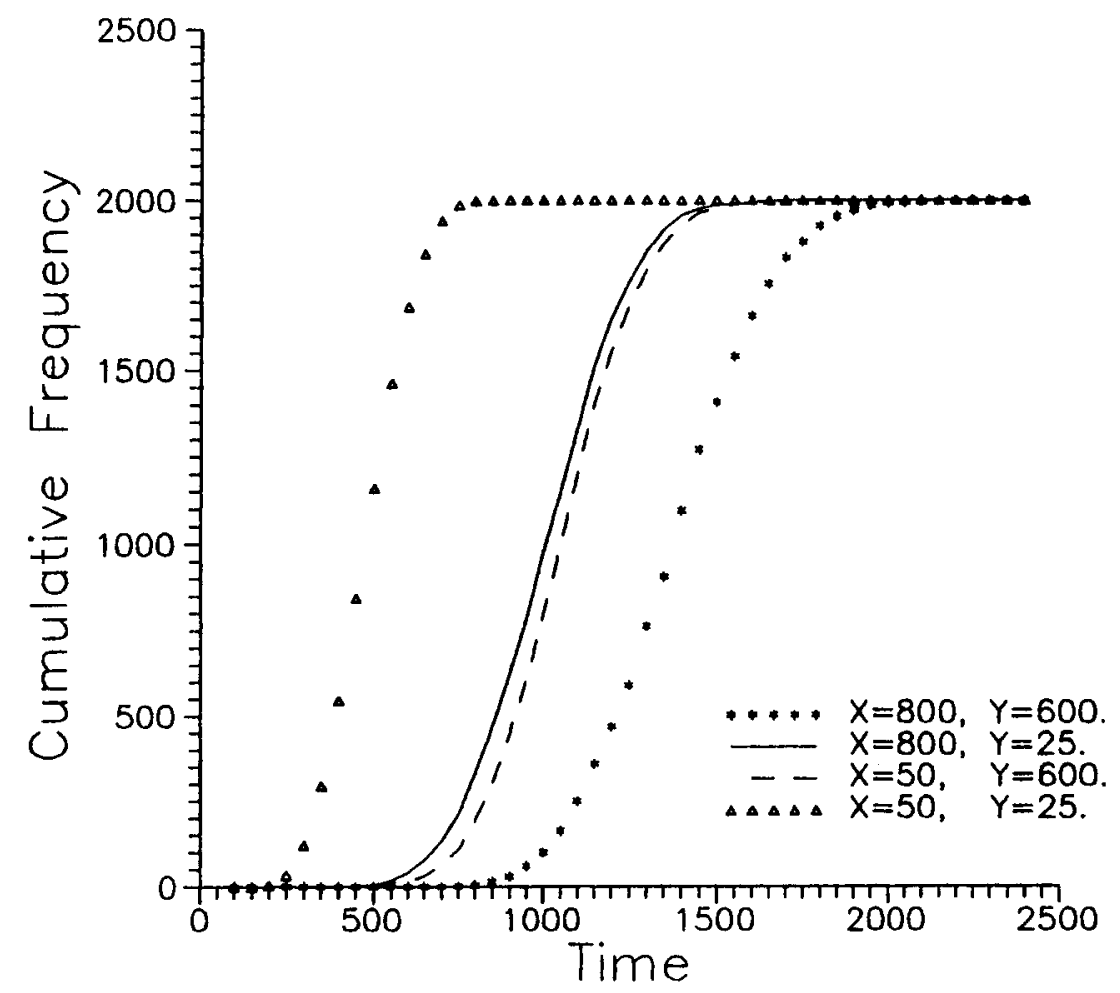

Figure 12. Simulated response time cumulative distribution functions (cdfs), as in Figure 10, but prolongations of $x$ and $y$ are relatively large.

For example, the means and standard deviations for the simulated response times for $x$ and $y$ in the Wheatstone bridge are shown in Table 3 . The factors have negative interactions. This prediction is satisfied by the means in the table for both $2 \times 2$ subdesigns alike.

If mean response times alone are considered, the interaction contrast has the same sign for concurrent processes and for processes on opposite sides of a Wheatstone bridge. This is true for both AND networks and OR networks. Although the sign of the mean interaction contrast is not informative, its magnitude is. In the case of the Wheatstone bridge, the magnitude of the interaction contrast for means converges as the factor levels increase (leading to stochastically slower processes) (Schweickert et al., 1992). Checking for this convergence is costly, however, because it requires a precise experiment with many factor levels.

On the other hand, our simulations demonstrate that it is quite feasible with an experiment of moderate size to distinguish the two cases using cdf contrasts. The key characteristics are shown in Table 4. The essential requirement for distinguishing process arrangements is that if a certain behavior can occur, it must be made to occur.

Even with cdf contrasts, sequential processes in a Wheatstone bridge cannot be distinguished from concurrent processes if the experiment uses only relatively low levels of the factors-that is, those corresponding to stochastically fast processes. The two arrangements can be distinguished only if a large range of levels of the factors is used.

Sequential processes in a serial-parallel network with AND gates cannot produce cdf contrasts that have positive regions, but no negative regions. True concurrent processes cannot lead to changes in sign (Schweickert et al., in press). Therefore, within the class of directed acyclic networks with AND gates, an experiment with several levels of the factors leading to the pattern shown in Figure 13 for low levels and to the pattern shown in Figure 14 for high levels rules out a serial-parallel network, and is the indication of a Wheatstone bridge. Similar reasoning would apply for networks with OR gates.

Another potential source of misclassification is that the Wheatstone bridge network in Figure 5 has AND gates, but it produces a property predicted for networks with OR gates. Specifically, the area under the interaction contrast in Figure 14 is zero or positive when measured from 0 to any time $t$; this is the property predicted for sequential processes in serial-parallel networks with OR gates (Schweickert et al., in press). But, as we earlier remarked, one can determine whether the gates are AND gates or OR gates by observing where changes in factor levels have effects on response time and where they do not.

The simulations in this section demonstrate that sequential processes on opposite sides of a Wheatstone bridge can behave, over a limited range, like either concurrent or sequential processes in a serial-parallel net- 


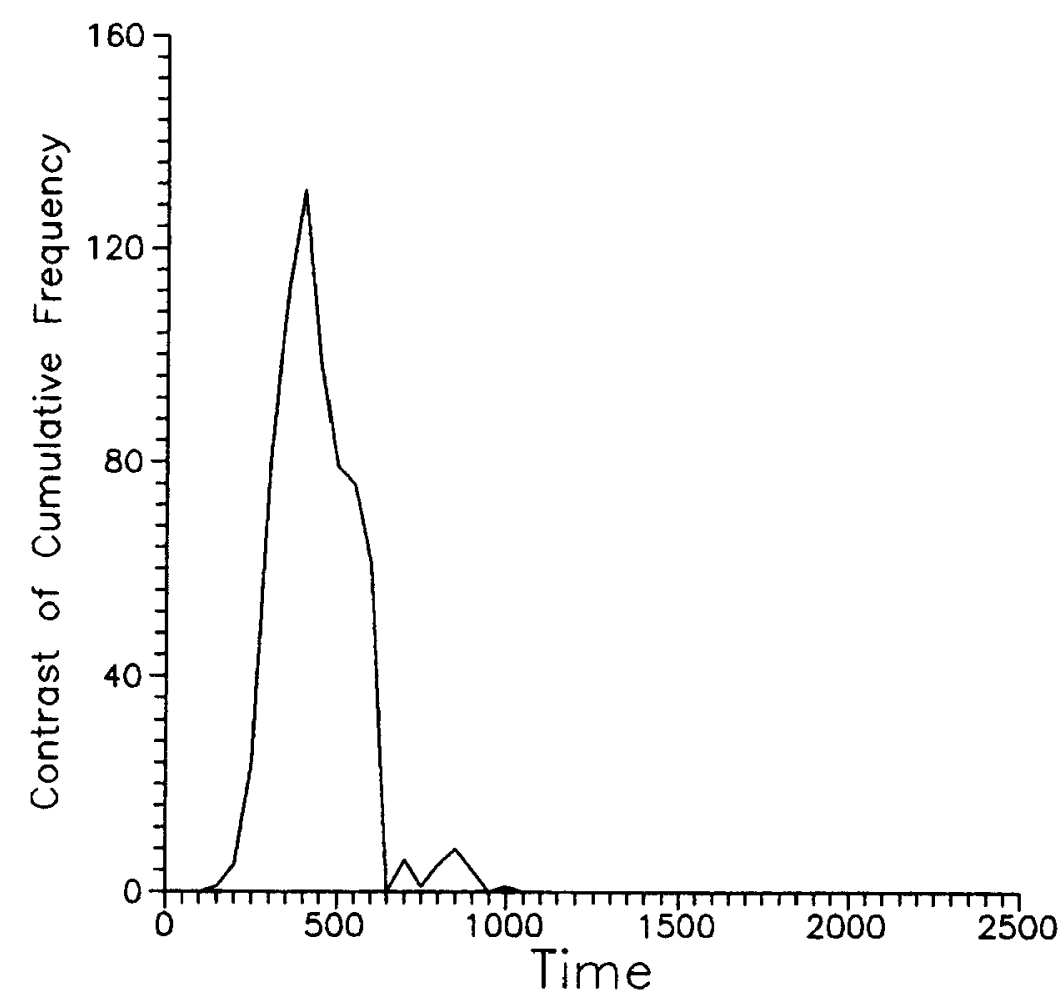

Figure 13. Simulated cumulative distribution function (cdf) contrast for the sequential processes $x$ and $y$ in a Wheatstone bridge. Prolongations of $x$ and $y$ are relatively small.

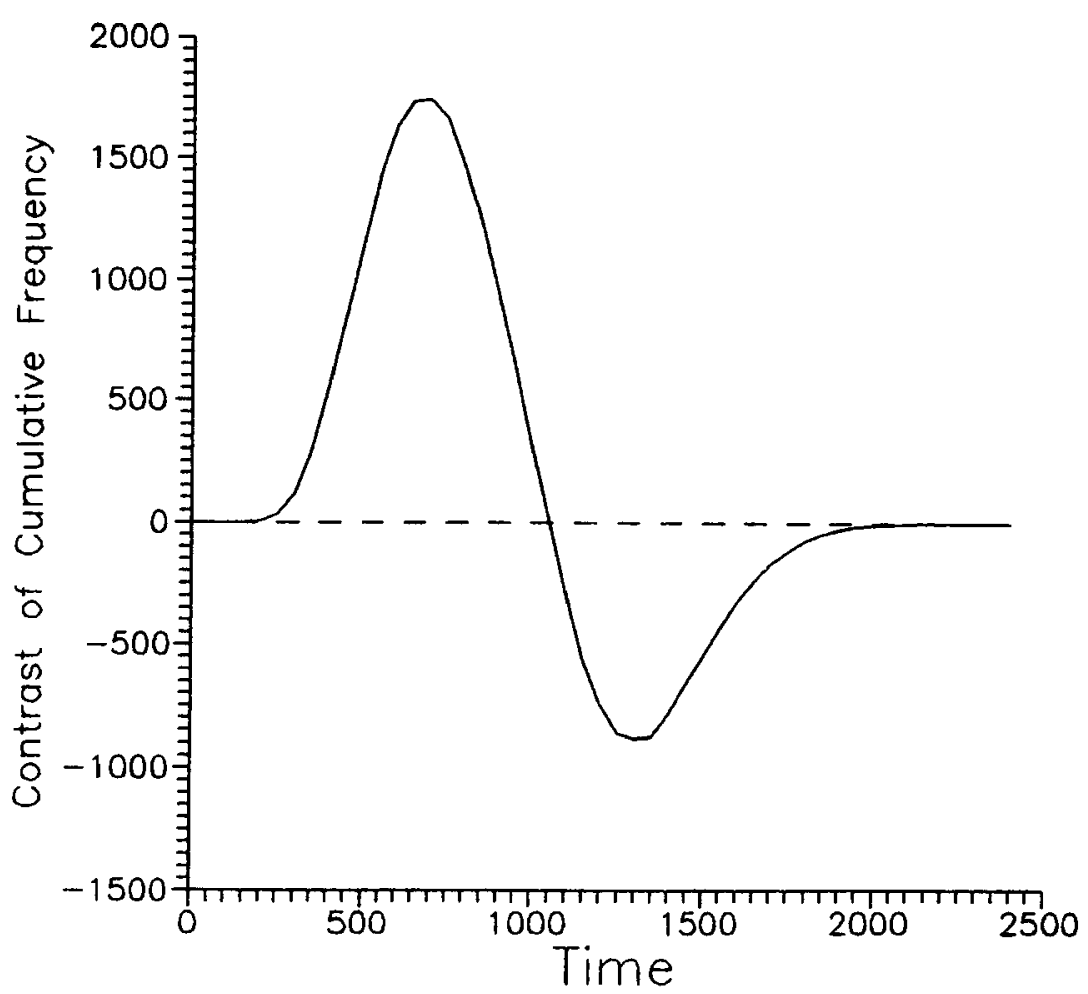

Figure 14. Simulated cumulative distribution function (cdf) contrast, as in Figure 11, but the prolongations of $x$ and $y$ are relatively large. 
work. The various cases can be distinguished by designing experiments having factors with a large range of levels.

\section{Statistical Remarks}

The tests described here were developed for graphical evaluation, and our simulations show that a feasible sample size can produce clearly visible patterns. In practice, of course, statistics may aid in reaching a conclusion.

For a given condition and given time $t$, let $G(t)=\pi$ be the cdf of the reaction time. Empirically, the cdf at a time $t$ is estimated as $P(t)=N(t) / N$, where $N(t)$ is the number of observations less than or equal to $t$ and $N$ is the total number of observations. The values of $N(t)$ follow a binomial distribution, with parameter $\pi$. Typically, there are hundreds or thousands of observations in a reaction time experiment, so the normal approximation to the binomial would be quite accurate. (Granted, there are few observations in the tails, but a cdf contrast at one of the tails would not usually be of interest.) Then $P(t)$ has approximately a normal distribution, with expected value $\pi$ and variance $\pi(1-\pi)$. These parameters can be estimated with $P(t)$ and $P(t)[1-P(t)]$, respectively. An estimate of the standard error of $P(t)$ is the square root of $P(t)[1-P(t)] / N$.

Now let $P_{i j}(t)$ be the empirical cdf when Factor $\mathrm{A}$ is at level $i$ and Factor B is at level $j$. Stochastic dominance at a time $t$ can be tested with the usual procedures for comparing two proportions (see, e.g., Hays, 1994; Kirk, 1990, pp. 416-419). The cdf contrast at a particular time $t$ is a linear combination of four approximately normal random variables, $P_{i j}(t)-P_{i 1}(t)-P_{1 j}(t)+P_{11}(t)$. The expected value of this contrast is $G_{i j}(t)-G_{i 1}(t)-G_{1 j}(t)+$ $G_{11}(t)$. Tests or confidence intervals for this contrast can be constructed in the usual way (e.g., based on Hays, 1994, Appendix C). If the experiment has a within-subjects design, each subject contributes reaction times in each condition. The cdf difference (or contrast) could be calculated for each subject individually. The mean of these differences (or contrasts) would then have, approximately, a $t$ distribution.

If tests or confidence intervals are needed at several times $t$, and these are widely spaced, the tests or intervals could be treated as independent. If the times are not widely spaced, the values of the empirical cdf at, say, $k$ points of time, will be dependent, and could be treated as having approximately a multivariate normal distribution.

\section{Summary}

Our simulations display conspicuous patterns in cdfs that can be used to classify processes. The qualitative predictions discussed here are easy to check graphically. Precise estimates of the cdfs are not required. On the other hand, the predictions are not designed for testing models on a quantitative basis, with, say, goodness-of-fit tests. Nor were they designed to be difficult to counterfeit, so no doubt there are models other than directed-acyclic networks that can make the same predictions.
However, for serial-parallel networks and the Wheatstone bridge, the predictions can distinguish among the various possibilities for process arrangements, provided a wide range of factor levels is used. The cdf interaction contrasts differ depending on whether the processes are concurrent or sequential and whether the gates are AND gates or OR gates (Nozawa, 1992; Schweickert et al., in press; Townsend \& Nozawa, 1995).

Our simulations show that there are possibilities for mimicking. Sequential processes in a Wheatstone bridge can mimic the patterns of both sequential and concurrent processes. However, with a wide range of factor levels, this arrangement can be identified through cdf interaction contrasts.

The additional information contained in the cdf interaction contrast predictions is obtained at a price; the assumptions underlying the tests of cdfs are stronger than those underlying the tests of means. The major additional assumptions are that (1) process durations are stochastically independent, and (2) factors selectively influencing processes produce stochastic dominance of the process durations. These are relatively strong assumptions, but they are supported indirectly in a variety of tasks.

The predictions for cdf contrasts are more stringent than are qualitative predictions previously derived for mean contrasts. The magnitude of the mean interaction contrast is simply the net area bounded by the cdf interaction contrast, while the algebraic sign of the former is the opposite of that of the latter. It is quite possible for the area under a cdf contrast to have the algebraic sign or the magnitude predicted for a certain process arrangement, while the curve itself does not have the form predicted for that arrangement. Therefore, cdfs can be used to falsify a model that means alone would not have falsified.

\section{REFERENCES}

Ashby, F. G., Tein, J. Y., \& Balakrishnan, J. D. (1993). Response time distributions in memory scanning. Journal of Mathematical Psychology, 37, 526-555.

Ashby, F. G., \& Townsend, J. T. (1980). Decomposing the reaction time distribution: Pure insertion and selective influence revisited. Journal of Mathematical Psychology, 21, 93-123.

Backus, B. T., \& Sternberg, S. (1988, November). Attentional tradeoff across space early in visual processing: New evidence. Paper presented at the annual meeting of the Psychonomic Society, Chicago.

Balakrishnan, J. D. (1994). Simple additivity of stochastic psychological processes: Tests and measures. Psychometrika, 59, 217-240.

Carrier, L. M., \& PAshler, H. (1995). Attentional limits in memory retrieval. Journal of Experimental Psychology: Learning, Memory, \& Cog:-ition, 21, 1339-1348.

Chechile, R. A. (1997, August). A new goodness-of-fit metric for interval data: A more powerful test of stochastic processes. Paper presented at the annual meeting of the Society for Mathematical Psychology, Bloomington, IN.

Cinlar, E. (1975). Introduction to stochastic processes. Englewood Cliffs, NJ: Prentice-Hall.

Coles, M. G. H., Smid, H. G. O. M., Scheffers, M. K., \& Otten, L. J. (1995). Mental chronometry and the study of human information processing. In M. D. Rugg \& M. G. H. Coles (Eds.), Electrophysiology of mind: Event-related brain potentials and cognition (pp. 86131). Oxford: Oxford University Press. 
Colonius, H., \& VorberG, D. (1994). Distribution inequalities for parallel models with unlimited capacity. Journal of Mathematical Psychology, 38, 35-58.

Cortese, J. M., \& Dzhafarov, E. N. (1995). Empirical recovery of response time decomposition rules II: Discriminability of serial and parallel architectures. Journal of Mathematical Psychology, 40, 185202.

DAvIS, R. (1957). The human operator as a single channel information system. Quarterly Journal of Experimental Psychology, 9, 119-129.

DE JONG, R. (1993). Multiple bottlenecks in overlapping task performance. Journal of Experimental Psychology: Human Perception \& Performance, 19, 965-980.

Dooin, B. (1985). Reducibility of stochastic networks. Omega International Journal of Management Science, 13, 223-232.

Dzhafarov, E. N. (1992). The structure of simple reaction time to stepfunction signals. Journal of Mathematical Psychology, 36, 235-268

Dzhafarov, E. N. (1993). Grice-representability of response time distribution families. Psychometrika, 58, 281-314.

Dzhafarov, E. N. (1997). Process representations and decompositions of response times. In A. A. J. Marley (Ed.), Choice, decision and measurement: Essays in honor of R. Duncan Luce (pp. 255-277). Hillsdale, NJ: Erlbaum.

Dzhafarov, E. N., \& Cortese, J. M. (1995). Empirical recovery of response time decomposition rules I: Sample-level decomposition tests. Journal of Mathematical Psychology, 40, 185-202.

Dzhafarov, E. N., \& Rouder, J. N. (1996). Empirical discriminability of two models for stochastic relationship between additive components of response time. Journal of Mathematical Psychology, $\mathbf{4 0}$ 48-63.

Dzhafarov, E. N., \& Schweickert, R. (1995). Decomposition of response times: An almost general theory. Journal of Mathematical Psychology, 39, 285-314.

Egeth, H., \& Dagenbach, D. (1991). Parallel versus serial processing in visual search: Further evidence from subadditive effects of visual quality. Journal of Experimental Psychology: Human Perception \& Performance, 17, 551-560.

Ehrenstein, A., Schweickert, R., Choi, S., \& Proctor, R. W. (1997). Scheduling processes in working memory: Instructions control the order of memory search and mental arithmetic. Quarterly Journal of Experimental Psychology, 50A, 766-802.

FISHER, D. L. (1985). Network models of reaction time: The generalized OP diagram. In G. d'Ydewalle (Ed.), Cognition, information processing and motivation (Vol. 3, pp. 229-254). Amsterdam: NorthHolland.

Fisher, D. L., \& Glasser, R. A. (1996). Molar and latent models of cognitive slowing: Implications for aging, dementia, depression, development, and intelligence. Psychonomic Bulletin \& Review, 3, 458-480.

Fisher, D. L., \& GoldsTeIN, W. M. (1983). Stochastic PERT networks as models of cognition: Derivation of the mean, variance, and distribution of reaction time using order-of-processing (OP) diagrams. Journal of Mathematical Psychology, 27, 121-151.

GoldSTEIN, W. M., \& FISHER, D. L. (1991). Stochastic networks as models of cognition: Derivation of response time distributions using the order-of-processing method. Journal of Mathematical Psychology, 35, 214-241.

GoldSTEIN, W. M., \& Fisher, D. L. (1992). Stochastic networks as models of cognition: Deriving predictions for resource-constrained mental processing. Journal of Mathematical Psychology, 36, 129-145.

GrICE, G. R. (1968). Stimulus intensity and response evocation. Psychological Review, 75, 359-373.

GRICE, G. R. (1970). Application of a variable criterion model to auditory reaction time as a function of the type of catch trial. Perception \& Psychophysics, 12, 103-107.

HaRTLY, H. O., \& Wortham, A. W. (1966). A statistical theory for PERT critical path analysis. Management Science, 12, B-469-B-481.

HAYS, W. L. (1994). Statistics (5th ed.). New York: Holt, Rinehart \& Winston

Johnston, J. C., MCCAnN, R. S., \& Remington, R. W. (1995). Chronometric evidence for two types of attention. Psychological Science, $\mathbf{6}$, 365-369
KaERKES, R., \& Mohring, R. H. (1978). Voresungen über Ordnungen und Netzplantheorie [Lectures on orders and a theory of networks] Aachen, Germany: Technischen Universität Aachen.

KIRK, R. E. (1990). Statistics: An introduction (3rd ed.). Fort Worth: Holt, Rinehart \& Winston.

Kounios, J. (1993). Process complexity in semantic memory. Journal of Experimental Psychology: Learning, Memory, \& Cognition, 19 , $338-351$.

Kounios, J., Osman, A. M., \& MEyer, D. E.. (1987). Structure and process in semantic memory: New evidence based on speed-accuracy decomposition. Journal of Experimental Psychology: General, 116, 3-25

LIU, Y. (1996). Queueing network model of elementary mental processes. Psychological Review, 103, 116-136.

LIVELY, B. L. (1972). Speed/accuracy trade-off and practice as determinants of stage durations in a memory-search task. Journal of Experimental Psychology, 96, 97-103.

LOGAN, G. D., \& BURKell, J. (1986). Dependence and independence in responding to double stimulation: A comparison of stop, change, and dual-task paradigms. Journal of Experimental Psychology: Human Perception \& Performance, 12, 549-563.

LuCE, R. D. (1986). Response times: Their role in inferring elementary mental organization. New York: Oxford University Press.

Marley, A. A. J., \& Colonius, H. (1992). The "horse race" random utility model for choice probabilities and reaction times, and its competing risks interpretation. Journal of Mathematical Psychology, 36, $1-20$.

MaSSARO, D. W., \& CoWAN, N. (1993). Information processing models: Microscopes of the mind. In L. W. Porter \& M. R. Rosenzweig (Eds.), Annual review of psychology (pp. 383-425). Palo Alto: Annual Reviews.

MCCANN, R. S., \& Johnston, J. C. (1992). Locus of the single-channel bottleneck in dual-task interference. Journal of Experimental Psychology: Human Perception \& Performance, 18, 474-484.

MeYer, D. E., \& KIERAS, D. E. (1997a). A computational theory of executive cognitive processes and multiple-task performance: Part 1 Basic mechanisms. Psychological Review, 104, 3-65.

Meyer, D. E., \& Kieras, E. E. (1997b). A computational theory of executive cognitive processes and multiple-task performance: Part 2. Accounts of psychological refractory-period phenomena. Psychological Review, 104, 749-791.

Meyer, D. E., Yantis, S., Osman, A., \& Smith, J. E. K. (1984). Discrete versus continuous models of response preparation: A reaction time analysis. In S. Kornblum \& J. Requin (Eds.), Preparatory states and processes (pp. 69-94). Hillsdale, NJ: Erlbaum

Micro ANALYsis and DESIGN (1985). MICROSAINT [Computer program]. Concord, MA: MGA, Inc.

MiLler, J. (1982). Divided attention: Evidence for coactivation with redundant signals. Cognitive Psychology, 14, 247-279.

NozaWA, G. (1992). Stochastic models of human information processing. Unpublished doctoral dissertation, Dartmouth College, Hanover, $\mathrm{NH}$

Osman, A., \& Moore, C. M. (1993). The locus of dual-task interference: Psychological refractory effects on movement-related brain potentials. Journal of Experimental Psychology: Human Perception \& Performance, 19, 1-21.

PASHLER, H. (1998). The psychology of attention. Cambridge, MA MIT Press.

Pashler, H., \& Johnston, J. C. (1989). Chronometric evidence for central postponement in temporally overlapping tasks. Quarterly Journal of Experimental Psychology, 41 A, 19-45.

ROBERTS, S., \& STERNBERG, S. (1993). The meaning of additive reactiontime effects: Tests of three alternatives. In D. E. Meyer \& S. Kornblum (Eds.), Attention and performance XIV: Synergies in experimental psychology, artificial intelligence, and cognitive neuroscience (pp. 611-654). Cambridge, MA: MIT Press.

Ruthruff, E., Miller, J., \& LachmanN, T. (1995). Does mental rotation require central mechanisms? Journal of Experimental Psychology: Human Perception \& Performance, 21, 552-570.

SANDERS, A. F. (1990). Issues and trends in the debate on discrete ver- 
sus continuous processing of information. Acta Psychologica, 74, 123-167.

Schuberth, R. E., Spoehr, K. T., \& Lane, D. M. (1981). Effects of stimulus and contextual information on the lexical decision process. Memory \& Cognition, 9, 68-77.

Schwartz, S. P., Pomerantz, J. R., \& Egeth, H. (1977). State and process limitations in information processing: An additive factor analysis. Journal of Experimental Psychology: Human Perception \& Performance, 3, 402-410.

SCHWEICKERT, R. (1978). A critical path generalization of the additive factor method: Analysis of a Stroop task. Journal of Mathematical Psychology, 18, 105-139.

SCHWEICKERT, R. (1982). Bias of an estimate of coupled slack in stochastic PERT networks. Journal of Mathematical Psychology, 26, 112.

ScHWEICKERT, R. (1983a). Latent network theory: Scheduling of processes in sentence verification and the Stroop effect. Journal of Experimental Psychology: Learning, Memory, \& Cognition, 9, 353383.

SCHWEICKERT, R. (1983b). Synthesizing partial orders given comparability information: Partitive sets and slack in critical path networks. Journal of Mathematical Psychology, 27, 261-276.

SCHWEICKERT, R. (1985). Separable effects of factors on speed and accuracy: Memory scanning, lexical decision, and choice tasks. $P s y$ chological Bulletin, 97, 530-546.

SCHWEICKERT, R. (1993). Information, time, and the structure of mental events: A 25-year review. In D. E. Meyer and S. Kornblum (Eds.), Attention and performance XIV: Synergies in experimental psychology, artificial intelligence, and cognitive neuroscience (pp. 535-566). Cambridge, MA: MIT Press.

SCHWEICKERT, R., \& BogGS, G. J. (1984). Models of central capacity and concurrency. Journal of Mathematical Psychology, 28, 223-281.

SCHWeickert, R., Fisher, D. L., \& GoldsteIN, W. M. (1992). General latent network theory: Structural and quantitative analysis of networks of cognitive processes (Tech. Rep. No. 92-1). West Lafayette, IN: Purdue University Mathematical Psychology Program.

Schweickert, R., Giorgini, M., \& Dzhafarov, E. (in press). Selective influence and response time cumulative distribution functions in serial-parallel task networks. Journal of Mathematical Psychology.

SchWEICKERT, R., \& TownSEND, J. T. (1989). A trichotomy: Interactions of factors prolonging sequential and concurrent mental processes in stochastic discrete mental (PERT) networks. Journal of Mathematical Psychology, 33, 328-347.

SCHWEICKERT, R., \& WANG, Z. (1993). Effects on response time of factors selectively influencing processes in acyclic task networks with OR gates. British Journal of Mathematical \& Statistical Psychology, 46, $1-40$.

Shaked, M., \& Shanthikumar, J. G. (1994). Stochastic orders and their applications. Boston: Academic Press.

STERNBERG, S. (1969). The discovery of processing stages: Extensions of Donders' method. In W. G. Koster (Ed.), Attention and performance II, (pp. 276-315). Amsterdam: North-Holland.

STOYAN, D. (1983). Comparison methods for queues and other stochastic models. New York: Wiley.

TOWNSEND, J. T. (1971). A note on the identifiability of parallel and serial processes. Perception \& Psychophysics, 10, 161-163.

TOWNSEND, J. T. (1976). Serial and within-stage independent parallel model equivalence on the minimum completion time. Journal of Mathematical Psychology, 14, 219-238.

TOWNSEND, J. T. (1990a). Serial vs. parallel processing: Sometimes they look like tweeledum and tweedledee but they can (and should) be distinguished. Psychological Science, 1, 46-54.

TOWNSEND, J. T. (1990b). Truth and consequences of ordinal differences in statistical distributions: Toward a theory of hierarchical inference. Psychological Bulletin, 108, 551-567.

TownSEND, J. T., \& AsHBy, F. G. (1983). Stochastic modeling of elementary psychological processes. Cambridge: Cambridge University Press.

Townsend, J. T., \& NozaWA, G. (1995). Spatio-temporal properties of elementary perception: An investigation of parallel, serial, and coactive theories. Journal of Mathematical Psychology, 39, 321-359.
VAN SELST, M., \& Jolicoeur, P. (1994). Can mental rotation occur before the dual-task bottleneck? Journal of Experimental Psychology: Human Perception \& Performance, 20, 905-921.

VAN ZANDT, T., \& RATCLIFF, R. (1995). Statistical mimicking of reaction time data: Single-process models, parameter variability, and mixtures. Psychonomic Bulletin \& Review, 2, 20-54.

WELFORD, A. T. (1952). The "psychological refractory period" and the timing of high-speed performance_a review and a theory. British Journal of Psychology, 43, 2-19.

\section{NOTE}

1. The predicted effects on mean reaction time of two factors selectively influencing two parallel processes are these: With AND gates, the combined effect of prolonging two processes is less than the sum of the effects of prolonging the processes separately. With OR gates, the combined effect is greater. For more details, and qualifying remarks, see Egeth and Dagenbach (1991), Nozawa (1992), Schweickert (1978), Schweickert and Wang (1993), and Townsend and Nozawa (1995).

\section{APPENDIX \\ Effects of Selectively Influencing Pairs of Processes in a Wheatstone Bridge}

In this first section of the Appendix we show that when pairs of processes in the Wheatstone bridge shown in Figure 5 are selectively influenced, the patterns predicted for the cdf interaction contrasts are the same as those predicted for pairs of processes in a serial-parallel network, except for the sequential processes on opposite sides of the "bridge" - that is, processes $x$ and $y$ in Figure 5. Processes $x$ and $y$ do not behave as sequential processes in a serial-parallel network, as demonstrated by our simulations.

Results in this section are based on a lemma from Schweickert et al. (in press), stated here in a slightly less general way.

Lemma. Suppose $r(u)$ and $s(u)$ are functions taking real numbers as arguments. Suppose there is a real $M$ such that $0 \leq$ $s(u) \leq M$ for all $u$.

(a) Suppose for all $t \geq 0$,

$$
\int_{t}^{\infty} r(u) d u \leq 0,
$$

and $s$ is a monotonically increasing function. Then for all $t>0$,

$$
\int_{i}^{\infty} r(u) s(u) d u \leq 0 .
$$

(b) Suppose for all $t>0$

$$
\int_{0}^{t} r(u) d u \geq 0,
$$

and $s$ is a monotonically decreasing function. Then for all $t>0$,

$$
\int_{0}^{t} r(u) s(u) d u \geq 0 .
$$

Notation. Let the duration of a process $x$ be the random variable $\mathrm{X}$. A value $\mathrm{X}$ takes on will be denoted $x$; it will be clear from context whether $x$ is the label for a process or the duration of a process. The density function for the duration of $x$ is assumed to exist. It is denoted $f_{x}(x)$ and the cdf is $F_{x}(x)$. The cdf for the response time is denoted $G(t)$.

If the process durations are stochastically independent, then the cdf for the response time in the Wheatstone bridge network of Figure 5, with AND gates, is 


$$
\begin{aligned}
G_{\mathrm{AND}}(t) & =\int_{0}^{1} \int_{0}^{1} F_{a}(t-x) F_{b}(t-x-y) F_{c}(t-y) d F_{x}(x) d F_{y}(y) \\
& =\int_{0}^{\infty} \int_{0}^{\infty} F_{a}(t-x) F_{b}(t-x-y) F_{c}(t-y) f_{x}(x) f_{y}(y) d x d y
\end{aligned}
$$

(Hartley \& Wortham, 1966).

Likewise, with OR gates, the survivor function of the response time is

$$
\begin{aligned}
\bar{G}_{\mathrm{OR}}(t) & =\int_{0}^{1} \int_{0}^{1} \bar{F}_{a}(t-x) \bar{F}_{b}(t-x-y) \bar{F}_{c}(t-y) d \bar{F}_{x}(x) d \bar{F}_{y}(y) \\
& =\int_{0}^{\infty} \int_{0}^{\infty} \bar{F}_{a}(t-x) \bar{F}_{b}(t-x-y) \bar{F}_{c}(t-y) f_{x}(x) f_{y}(y) d x d y .
\end{aligned}
$$

Because $d \bar{F}_{x}(x)=-d F_{x}(x)$ and $d \bar{F}_{y}(y)=-d F_{y}(y)$, the product of differentials in the above equation can be written $d F_{x}(x)$ $d F_{y}(y)=f x(x) f y(y) d x d y$.

In, what follows, we derive expressions for AND gates explicitly, leaving expressions for OR gates to the reader. To simplify the notation, we will omit the subscripts AND and OR.

It follows immediately from Equation $A 1$ that if a factor selectively influences one of the processes in the Wheatstone bridge, producing stochastic dominance for the process duration, then stochastic dominance is produced for the response times. Consider, for example, a factor selectively influencing process $a$. Let $F_{a 1}(a)$ be the cdf for the duration of $a$ when the factor is at Level 1 , and let $F_{a 2}(a)$ be the cdf when the factor is at Level 2. Let the corresponding cdfs for the response times be denoted $G_{1}(t)$ and $G_{2}(t)$. It can be seen immediately from Equation A1 that if for all $a, F_{a 1}(a) \geq F_{a 2}(a)$, then for all $t, G_{1}(t) \geq G_{2}(t)$. The reasoning is similar for processes $b$ and $c$.

Turning now to process $x$, suppose a factor selectively influences $x$ so the duration of $x$ at Level 1 of the factor is stochastically faster than that at Level 2 of the factor. Then

$$
\int_{0}^{t} f_{x 1}(x)-f_{x 2}(x) d x=F_{x 1}(t)-F_{x 2}(t) \geq 0
$$

for all $t$. Let

$$
s(x)=\int_{0}^{\infty} F_{a}(t-x) F_{b}(t-x-y) F_{c}(t-y) f_{y}(y) d y .
$$

Note that for a fixed $t, s(x)$ is monotonically decreasing in $x$ and $0 \leq s(x) \leq 1$. Then by the lemma,

$$
\begin{aligned}
0 & \leq \int_{0}^{t} s(x)\left[f_{x 1}(x)-f_{x 2}(x)\right] d x \\
& =\int_{0}^{t} \int_{0}^{\infty} F_{a}(t-x) F_{b}(t-x-y) F_{c}(t-y) f_{y}(y) d y\left[f_{x 1}(x)-f_{x 2}(x)\right] d x \\
& =G_{1}(t)-G_{2}(t) .
\end{aligned}
$$

Then the factor selectively influencing $x$ produces stochastic dominance for the response times. [Because $F_{a}(t-x)=0$ for $x \geq t$, it does not matter whether the upper limit on the first integral is $t$ or $\infty$.] The reasoning for a factor selectively influencing $y$ is similar.
We now consider all possible pairs of the five processes in the Wheatstone bridge illustrated in Figure 5, except for $x$ and $y$. We consider five concurrent pairs and four sequential pairs.

\section{The Concurrent Pairs of Processes}

In this section we will show that every pair of concurrent processes in the Wheatstone bridge behaves as a pair of concurrent processes in a serial-parallel network; that is, the cdf interaction contrast is nonnegative for all times $t$.

For the processes $a$ and $b$, suppose $F_{a 1}(a) \geq F_{a 2}(a)$ for all $a$ and $F_{b 1}(b) \geq F_{b 1}(b)$ for all $b$. Then for all $t$,

$$
\begin{aligned}
c(t)= & G_{11}(t)-G_{12}(t)-G_{21}(t)+G_{22}(t) \\
= & \int_{0}^{\infty} \int_{0}^{\infty}\left[F_{a 1}(t-x)-F_{a 2}(t-x)\right]\left[F_{b 1}(t-x-y)-F_{b 2}(t-x-y)\right] \\
& F_{c}(t-y) f_{x}(x) f_{y}(y) d x d y \geq 0 .
\end{aligned}
$$

A similar argument shows $c(t) \geq 0$ for all $t$ for the pairs of processes $\{b, c\}$ and $\{a, c\}$.

Consider the concurrent processes $x$ and $c$. For a fixed $x$ and $t$ let

$$
s(x, t)=\int_{0}^{\infty} F_{a}(t-x) F_{b}(t-x-y)\left[F_{c 1}(t-y)-F_{c 2}(t-y)\right] f_{y}(y) d y .
$$

For a fixed $t, s(x, t)$ is monotonically decreasing in $x$. Further, because $F_{c 1}(t-y)-F_{c 2}(t-y) \geq 0, s(x, t) \geq 0$. Finally,

$$
\begin{aligned}
s(x, t) & \leq \int_{0}^{\infty} F_{a}(t-x) F_{b}(t-x-y) F_{c 1}(t-y) f_{y}(y) d y \\
& \leq \int_{0}^{\infty} f_{y}(y) d y=1 .
\end{aligned}
$$

Hence, from Equation A2 and the lemma,

$$
c(t)=\int_{0}^{t} s(x, t)\left[f_{x 1}(x)-f_{x 2}(x)\right] d x \geq 0 .
$$

Similar reasoning shows that $\mathrm{c}(t) \geq 0$ for all $t$ for the concurrent pair $\{a, y\}$.

We have now discussed all possible pairs of concurrent processes in the Wheatstone bridge of Figure 5, so we turn to the sequential pairs.

\section{The Sequential Pairs of Processes}

We begin by describing the behavior of the cdf interaction contrast at times slightly greater than zero. Then we turn to its behavior over longer time intervals.

Times near zero. The first result, essentially, is that for sequential processes in the Wheatstone bridge, the cdf interaction contrast at small values of $t$ is positive if the gates are AND gates, negative for $x$ and $y$ if the gates are OR gates, and positive for all other sequential pairs if the gates are OR gates.

As before, we assume that a factor selectively influencing a process produces stochastic dominance for the cdfs of the process duration. In addition, if one of the influenced processes is $x$, we assume there is a time $\tau_{x}$ such that $f_{x 1}(x) \geq f_{x 2}(x)$ for $0 \leq$ $x \leq \tau_{x}$. Likewise, if one of the influenced processes is $y$, we assume there is a time $\tau_{y}$ such that $f_{y 1}(y)-f_{y 2}(y) \geq 0$ for $0 \leq y \leq \tau_{y}$.

Suppose the sequential processes $x$ and $a$ are selectively influenced by a pair of experimental factors. Suppose the gates are AND gates. When the factor selectively influencing $x$ is at 
level $i$, and the factor selectively influencing $a$ is at level $j$, the response time cdf can be written

$$
G_{i j}(t)=\int_{0}^{\infty} \int_{0}^{\infty} F_{a j}(a) F_{b}(a-y) F_{c}(t-y) f_{x i}(t-a) f_{y}(y) d a d y .
$$

This equation is obtained from Equation Al by the change of variables $a=t-x$. The cdf interaction contrast is

$$
\begin{aligned}
c(t)=\int_{0}^{\infty} \int_{0}^{\infty}\left[F_{a 1}(a)-F_{a 2}(a)\right] F_{b}(a-y) F_{c}(t-y) \\
\\
{\left[f_{x 1}(t-a)-f_{x 2}(t-a)\right] f_{y}(y) d a d y . }
\end{aligned}
$$

By the assumption of stochastic dominance, $F_{a 1}(a)-F_{a 2}(a) \geq 0$. By the additional assumption about the factor selectively influencing $x, f_{a 1}(t-a)-f_{a 2}(t-a) \geq 0$ for $0 \leq t \leq \tau_{x}$. Note that $f_{a 1}(t-a)-f_{a 2}(t-a)=0$ for $a \geq t$. It follows that $c(t) \geq 0$ for 0 $\leq t \leq \tau_{x}$. If the integrand is 0 throughout the interval $0 \leq t \leq \tau_{x}$, then $c(t)$ will be 0 throughout the interval. This is unlikely to occur in practice.

Similar reasoning shows that $c(t) \geq 0$ for small values of $t$ when the other pairs of sequential processes are prolonged. In particular, for the sequential pair $x$ and $y, c(t) \geq 0$ for $0 \leq t \leq$ $\min \left\{\tau_{x}, \tau_{y}\right\}$.

Finally, if the gates are OR gates, for the sequential pair $x$ and $y, c(t) \leq 0$ for small values of $t$. For all other pairs of sequential processes, $c(t) \geq 0$ for small values of $t$.

Extended time intervals. In this section, we consider the area bounded by the cdf interaction contrast, $c(t)$. As usual, we assume that a factor selectively influencing a process produces stochastic dominance for the cdfs of the process duration.

Suppose the gates are AND gates. If two sequential processes other than $x$ and $y$ are selectively influenced, then for all $t \geq 0$

$$
\int_{t}^{\infty} c(t) d t \leq 0
$$

To see this, consider, for example, the pair of processes $x$ and $a$. The cdf interaction contrast is given by Equation A3.

Note that

$$
\int_{t}^{\infty} f_{x 1}(t-a)-f_{x 2}(t-a) d t=\bar{F}_{x 1}(t-a)-\bar{F}_{x 2}(t-a) \leq 0 .
$$

Further, $0 \leq F_{c}(t-y) \leq 1$ and for a fixed $y, F_{c}(t-y)$ is monotonically increasing in $t$. Then by the lemma,

$$
\int_{t}^{\infty} F_{c}(t-y)\left[f_{x 1}(t-a)-f_{x 2}(t-a)\right] d t \leq 0 .
$$

By stochastic dominance, $F_{a 1}(a)-F_{a 2}(a) \geq 0$. Then

$$
\begin{aligned}
& {\left[F_{a 1}(a)-F_{a 2}(a)\right] F_{b}(a-y) \int_{t}^{\infty} F_{c}(t-y)} \\
& {\left[f_{x 1}(t-a)-f_{x 2}(t-a)\right] d t \leq 0}
\end{aligned}
$$

Therefore,

$$
\begin{aligned}
& \int_{t}^{\infty} c(t) d t \\
& =\int_{0}^{\infty} \int_{0}^{\infty}\left[F_{a 1}(a)-F_{a 2}(a)\right] F_{b}(a-y) \int_{t}^{\infty} F_{c}(t-y) \\
& \quad\left[f_{x 1}(t-a)-f_{x 2}(t-a)\right] d t d a d y \leq 0 .
\end{aligned}
$$

In a similar way, Equation A4 can be shown to hold for the sequential pairs $\{x, b\},\{y, c\}$, and $\{y, b\}$.

(Manuscript received May 24, 1996; revision accepted for publication July 14, 1998 .) 